Garrido, J., Vilar, L., Echavarría, P., Martínez-Vega, J, Martín M. P. (2018): “¿Pueden las interfaces de usos del suelo explicar la ocurrencia de incendios forestales a escala provincial? Los casos de Zamora y Madrid", GeoFocus (Artículos), no 22, p. 71-95. ISSN: 1578-5157 http://dx.doi.org/10.21138/GF.611

\title{
¿PUEDEN LAS INTERFACES DE USOS DEL SUELO EXPLICAR LA OCURRENCIA DE INCENDIOS FORESTALES A ESCALA PROVINCIAL? LOS CASOS DE ZAMORA Y MADRID
}

\author{
JESÚS GARRIDO $^{1 *}$, LARA VILAR $^{1}$, PILAR ECHAVARRÍA $^{1}$, JAVIER MARTÍNEZ-VEGA $^{1}$, \\ M. PILAR MARTÍN ${ }^{1}$ \\ ${ }^{1}$ Instituto de Economía, Geografía y Demografía, Centro de Ciencias Humanas y Sociales, \\ Consejo Superior de Investigaciones Científicas (CSIC), Albasanz 26-28, 28037, Madrid, \\ España. \\ *jesgarri93@gmail.com
}

RESUMEN

Los usos del suelo y su relación con factores socio-económicos son un aspecto clave en la gestión de incendios forestales. Este estudio analiza la ocurrencia de incendios forestales en el período 2005-2010 utilizando como variables explicativas las zonas de contacto entre usos del suelo (interfaces: agrícola-forestal (IAF), urbano-forestal (IUF) y pasto-forestal (IPF)) por su relación con la ocurrencia de incendios en España, calculadas a partir de mapas de usos del suelo de Climate Change Initiative-Land Cover. Se utilizó MaxEnt para generar dos modelos predictivos (general y específico) en Zamora y Madrid. En el modelo general, la IAF contribuyó más en Zamora y la IUF en Madrid. El modelo específico mejoró los ajustes generales en ambas provincias, los porcentajes de acierto en Zamora y la discriminación espacial del riesgo, optimizando las potenciales actuaciones dirigidas a la prevención.

Palabras clave: MaxEnt, incendio forestal, interfaz, usos del suelo, causalidad, riesgo

\section{¿PUEDEN LAS INTERFACES DE USOS DEL SUELO EXPLICAR LA OCURRENCIA DE INCENDIOS FORESTALES A ESCALA PROVINCIAL? LOS CASOS DE ZAMORA Y MADRID}

\section{ABSTRACT}

Land uses and their relationship with socio-economic factors are a key aspect in the management of wildfires. This study analyzes the forest fires occurrence in the period 20052010 using as explanatory variables the contact zones between land uses (interfaces: agricultural-forest (AFI), urban-forest (WUI) and grassland-forest (GFI)) due to its relationship with the wildfire occurrence in Spain, calculated from Climate Change Initiative-Land Cover maps, and using MaxEnt to generate two predictive models (general and specific) in Zamora 
Garrido, J., Vilar, L., Echavarría, P., Martínez-Vega, J, Martín M. P. (2018): “¿Pueden las interfaces de usos del suelo explicar la ocurrencia de incendios forestales a escala provincial? Los casos de Zamora y Madrid", GeoFocus (Artículos), no 22, p. 71-95. ISSN: 1578-5157 http://dx.doi.org/10.21138/GF.611

and Madrid. In the general model, the AFI contributed the more in Zamora, and the WUI in Madrid. The vegetation formations models generally improved the accuracy values in both provinces and the percentage of agreement in Zamora and the spatial risk discrimination, optimizing potential actions aimed at prevention.

Keywords: MaxEnt, wildfires, interface, land uses, fire incidence causes, risk.

\section{Introducción}

Los incendios forestales se producen como consecuencia de la existencia de agentes de ignición, de origen humano o natural, y su interacción con factores ambientales favorables a la combustión y propagación. Su tamaño y frecuencia se relacionan directamente con factores tales como la temperatura, humedad, viento y el tipo y características de la vegetación, entre otros. La ocurrencia de incendios puede verse notablemente influenciada por los patrones de usos y coberturas del suelo (Parisien y Moritz 2009). Las zonas climáticas templadas de latitudes medias presentan características que favorecen, en gran medida, la ocurrencia de incendios forestales, ya que constituyen un complejo medio ecológico entre climas extremos con suficiente cantidad de biomasa y condiciones meteorológicas favorables (Bond y van Wilgen 1996, van der Werf et al. 2003, Spessa et al. 2005). Las actividades humanas están en el origen de la mayoría de los incendios forestales que se producen en el mundo, principalmente en zonas donde distintos usos y coberturas forestales del suelo se encuentran muy entremezclados con otros usos agrícolas y urbanos. También en ecosistemas con coberturas del suelo más homogéneas como sabanas y bosques tropicales (Parisien y Moritz 2009).

En la cuenca mediterránea europea los incendios forestales ocasionan el $90 \%$ del total de pérdidas ambientales y económicas debidas a catástrofes naturales. Preocupan los impactos ambientales, ecológicos y socio-económicos que generan y su creciente intensidad y ocurrencia (Pérez-Cabello et al. 2009, Renard et al. 2012, Feng et al. 2015, Vilar et al. 2016). La acción del hombre está directa o indirectamente relacionada con el $85 \%$ de los incendios forestales que se producen en esta región (Ganteaume et al. 2013, Vilar et al. 2016).

La Europa Mediterránea ha sufrido, en el último siglo, un importante proceso de transformación socio-económica y territorial. Tras la Segunda Guerra Mundial, padeció un fuerte éxodo rural hacia las zonas urbanas, siendo más notable en las décadas de los años $50 \mathrm{y}$ 60 en países como Portugal, España o Italia. En los años 80 y 90, las zonas rurales de las regiones metropolitanas y litorales han sufrido una enorme transformación. El aumento de la presión urbana sobre el medio natural ha ocasionado nuevos desarrollos urbanísticos y la dispersión de segundas residencias (Camarero 1993, Romero-Calcerrada y Perry 2004, Millington et al. 2009, Vilar et al. 2016). Esta tendencia se ha prolongado durante el siglo XXI. Todas estas transformaciones socio-económicas han implicado cambios de usos del suelo que han tenido efecto directo sobre la ignición y la propagación de incendios (Romero-Calcerrada y Perry 2004, Millington et al. 2009, Bowman et al. 2011, Vilar et al. 2016). Se ha incrementado notablemente la superficie de la conocida como interfaz urbano-forestal, IUF (Radeloff et al. 2005), incrementando igualmente el riesgo de ignición por accidentes o negligencias. En las regiones rurales, a pesar de la modernización que las prácticas agrícolas han experimentado en las últimas décadas y de las restricciones, se sigue utilizando el fuego como herramienta 
Garrido, J., Vilar, L., Echavarría, P., Martínez-Vega, J, Martín M. P. (2018): “¿Pueden las interfaces de usos del suelo explicar la ocurrencia de incendios forestales a escala provincial? Los casos de Zamora y Madrid", GeoFocus (Artículos), no 22, p. 71-95. ISSN: 1578-5157 http://dx.doi.org/10.21138/GF.611

tradicional para crear nuevas zonas de pasto para el ganado y la agricultura, limpiar la maleza en los límites de las parcelas de cultivos o en las tierras abandonadas o para eliminar los residuos de las cosechas (Ganteaume et al. 2013, Vilar et al. 2016). Este hábito puede dar lugar a que el fuego se expanda de forma accidental, negligente o intencionada a las zonas naturales cercanas. No obstante, en las regiones rurales más periféricas, el éxodo rural ha dado lugar al abandono de las actividades tradicionales y de las tierras de cultivo, lo que ha provocado el crecimiento descontrolado y no gestionado de vegetación y, por tanto, la acumulación de combustible dando lugar a un incremento en el riesgo de incendios (Ganteaume et al. 2013). Por último, en todas las regiones, el incremento del número de visitantes a las zonas forestales se relaciona con un aumento del riesgo de incendio por accidentes o negligencias asociados al incremento de actividades de tipo recreativo (Ganteaume et al. 2013, Vilar et al. 2016). Lógicamente, este fenómeno es más intenso en las regiones urbanas y periurbanas.

España es uno de los países de la Europa Mediterránea más afectados por los incendios forestales, con una media de 17.000 incendios al año. En el decenio 2001-2010, se produjeron un total de 171.270 siniestros. La Comunidad Autónoma con mayor número de incendios fue Galicia (42\% de los siniestros), seguida de Castilla y León (11,2 \%) y Asturias (10,3\%). En el otro extremo, Canarias, Murcia, La Rioja, Islas Baleares y País Vasco no llegaron al $1 \%$. Al igual que en el resto de la cuenca mediterránea europea, el $90 \%$ de los incendios forestales en España son debidos a las actividades humanas (Nicolás y Caballero 2001, Vilar et al. 2016). Las principales causas asociadas a la ocurrencia difieren, sin embargo, a nivel regional. Así, en las zonas más rurales, como las provincias interiores de Castilla y León, Castilla-La Mancha o Extremadura, los incendios suelen estar asociados al uso imprudente del fuego en las labores agrícolas o son producidos intencionadamente, mientras que, en otras zonas más urbanizadas como Madrid, Valencia o Barcelona, la causalidad se relaciona, en mayor medida, con las negligencias y accidentes derivados de los usos turísticos y recreativos del territorio.

Estos procesos son críticos en las zonas de contacto entre las coberturas forestales, por un lado, y las agrícolas y urbanas, por otro. Las zonas de interfaz urbano-forestal han recibido especial atención a causa del incremento anual de superficie quemada y de las pérdidas humanas y económicas producidas (National Interagency Fire Center 2004, Syphard et al. 2008, LampinMaillet et al. 2011, Vilar et al. 2016). Su vigilancia es el mayor reto de cara a la protección de los bienes y las personas frente a los incendios forestales (Cohen 2000, Winter y Fried 2001, Bar Massada et al. 2009). Además, la interfaz agrícola-forestal tiene gran relevancia. Durante el período 2001-2010, las quemas agrícolas han sido la principal causa o motivación de todos los incendios intencionados (42,71\% del total, Cubo et al. 2012). Por último, durante el mismo periodo la IPF ha concentrado el 25,69\% de los incendios intencionados (Cubo et al. 2012).

Las interfaces se han considerado en trabajos anteriores como indicadores indirectos para el análisis del riesgo de incendio (Gallardo et al. 2015, Vilar et al. 2016). Además, se han empleado como variables para representar los factores socio-económicos. En la España peninsular, Rodrigues et al. (2014) han estudiado la influencia de las interfaces IUF e IAF, llegando a la conclusión de que, en relación a la ignición, la IAF tenía más importancia que la IUF y otras variables, debido al uso del fuego como herramienta de limpieza de los desechos agrícolas en gran parte de España. En Madrid, Gallardo et al. (2015) han cartografiado los cambios de usos del suelo ocurridos en las últimas décadas y simulado los cambios esperados en el patrón espacial de las interfaces, de acuerdo a distintos escenarios, con el objetivo de conocer su influencia sobre el riesgo de incendio. En este estudio las interfaces IAF e IUF representan espacialmente factores de cambio y causas antropogénicas de incendios, mejor que otras 
Garrido, J., Vilar, L., Echavarría, P., Martínez-Vega, J, Martín M. P. (2018): “¿Pueden las interfaces de usos del suelo explicar la ocurrencia de incendios forestales a escala provincial? Los casos de Zamora y Madrid", GeoFocus (Artículos), no 22, p. 71-95. ISSN: 1578-5157 http://dx.doi.org/10.21138/GF.611

variables empleadas en otros modelos a escala regional o global, tales como la accesibilidad o la densidad de población. En California, Syphard et al. (2008) han descubierto que la mayoría de los incendios estaban relacionados espacialmente, sobre todo, con la IUF y su área de influencia. A pesar de su importancia, pocos trabajos han abordado el desafío de dividir estas tres interfaces con objeto de refinar los modelos predictivos de riesgo.

Por último, basados en métodos y tecnologías diferentes, se han desarrollado distintos modelos predictivos del riesgo de incendio que facilitan la toma de decisiones de los gestores. Diversos autores han utilizado los Modelos Lineales Generalizados (GLM, por sus siglas en inglés, Generalized Linear Models (Nelder y Wedderburn 1972)) obteniendo resultados adecuados de predicción de ocurrencia de incendio. Incluyen variables de tipo socio-económico, en zonas de clima mediterráneo, tales como España (Martínez et al. 2009, Chuvieco et al. 2010) y California (Syphard et al. 2007, Syphard et al. 2008). Los métodos de presencia-ausencia (regresiones logísticas) pertenecen a los GLM y también han sido empleados para obtener predicciones de ocurrencia. Tienen la ventaja de poder considerar el habitual desequilibrio de ocurrencias (incendios) frente a no ocurrencias (ausencia de incendios), comunes en la modelización del riesgo de incendio forestal (Vilar et al. 2016). También han sido comúnmente empleados en estudios sobre riesgo de incendio forestal los algoritmos de aprendizaje automático (machine learning algorithms), tales como Random Forest (Bar Massada et al. 2012, Oliveira et al. 2012), Weights of Evidence (Romero-Calcerrada et al. 2008) y Classification Trees (Sturtevant y Cleland 2007), que presentan una importante ventaja: son modelos no paramétricos; por tanto, las interrelaciones de las variables independientes de entrada no son definidas en un primer estadio, sino que son resultado de las pruebas y muestreos, mediante la utilización de subconjuntos de datos aleatorios (Hastie et al. 2009).

Dentro de las herramientas de machine learning, el algoritmo de MaxEnt, el método de máxima entropía (presence-only Maximum Entropy (Phillips et al. 2006), ha sido creado para elaborar Modelos de Distribución Espacial (Spatial Distribution Models, SDM, sus siglas en inglés) de especies de flora y fauna, en relación a diversas variables ambientales (Phillips et al. 2006, Bar Massada et al. 2012). MaxEnt tiene mayor capacidad de predicción que otros SDM, sobre todo en muestras de tamaño pequeño (Elith et al. 2006, Pearson et al. 2007). MaxEnt ha sido empleado, por primera vez, en estudios ecológicos por Phillips et al. (2004) y aplicado, con éxito, como un instrumento para la modelización de la distribución de especies utilizando sólo los datos disponibles de la presencia de las especies (Couturier et al. 2014, Giovannini et al. 2014, Pena et al. 2014). Los principios que rigen la aplicación de MaxEnt a la distribución espacial de especies son, en principio, válidos para su uso en la estimación de la ocurrencia de incendios ya que su presencia y distribución territorial, está condicionada por diversas variables ambientales (Parisien y Moritz 2009). Varios autores han aplicado MaxEnt a la modelización de los factores medioambientales relacionados con la distribución espacial de incendios forestales (Parisien y Moritz 2009, Renard et al. 2012) a escalas espaciales muy diferentes. MaxEnt se ha utilizado para evaluar, de manera cuantitativa, los factores medioambientales de la distribución espacial de los puntos de ignición detectados por el sensor MODIS (Moderate-Resolution Imaging Spectroradiometer) en la costa occidental de India. Teniendo en cuenta sólo los lugares con presencia de incendios para modelizar los requisitos de idoneidad del fuego, estos autores han analizado los lugares que se habían quemado y aquellos donde se podría haber producido un fuego porque presentaban las características necesarias para que se produjera un incendio (Renard et al. 2012). MaxEnt ha mostrado mayor precisión en la predicción de ocurrencia de incendios que otros modelos estadísticos paramétricos como GLM u otros modelos de machine learning en trabajos relacionados con el análisis y comparación de la distribución de más de 200 


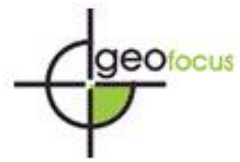

Garrido, J., Vilar, L., Echavarría, P., Martínez-Vega, J, Martín M. P. (2018): “¿Pueden las interfaces de usos del suelo explicar la ocurrencia de incendios forestales a escala provincial? Los casos de Zamora y Madrid", GeoFocus (Artículos), no 22, p. 71-95. ISSN: 1578-5157 http://dx.doi.org/10.21138/GF.611

especies de fauna y flora silvestres en distintos países, empleando más de 10 técnicas de modelado utilizando únicamente los datos de presencia de las especies (Elith et al. 2006, Guisan et al. 2007); o en el análisis de ocurrencia de incendios en Michigan (Estados Unidos) empleando tres tipos de modelos estadísticos, Bar Massada et al. (2012) concluyeron que MaxEnt y Random Forest se ajustaron mejor que GLM en términos generales de precisión.

El objetivo de este trabajo es demostrar la capacidad de las interfaces de usos de suelo para explicar y predecir la ocurrencia de incendios en España a escala regional, utilizando como áreas test dos provincias, Zamora y Madrid. Sus características socio-económicas, ambientales y de incidencia de incendios son muy contrastadas.

\section{Metodología}

\section{1. Áreas de estudio}

Este trabajo analiza dos zonas de estudio en España: las provincias de Zamora (Castilla y León) y Madrid (Comunidad de Madrid). El mapa de localización de las provincias, así como las coberturas del suelo de CCI-LC 2010, se muestran en la Figura 1.

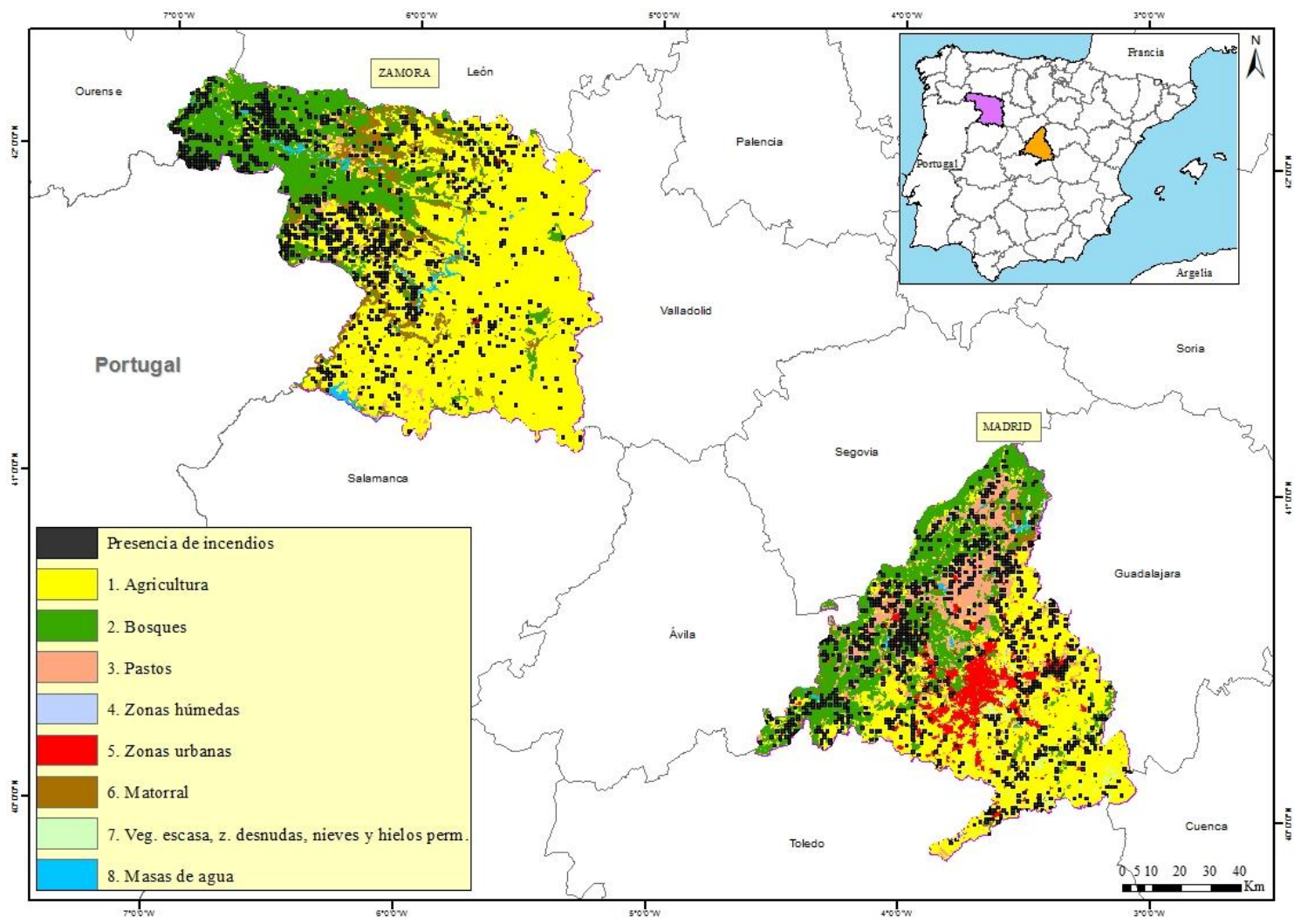

Figura 1. Localización de las provincias de estudio, coberturas del suelo de CCI-LC 2010 y puntos de ignición de incendio forestal (2005-2007 Zamora, 2005-2010 Madrid).

Zamora tiene una superficie superior a la de Madrid $\left(\sim 10.500 \mathrm{~km}^{2}\right.$ frente a $\left.\sim 8.000 \mathrm{~km}^{2}\right)$, aunque su número de habitantes y densidad media de población es significativamente menor (17 frente a $800 \mathrm{hab} / \mathrm{km}^{2}$; INE, 2018).

El clima mediterráneo continental es el predominante en las dos provincias, con unas temperaturas medias anuales que oscilan entre los $12-14^{\circ} \mathrm{C}$, con amplitudes térmicas de 15 - 


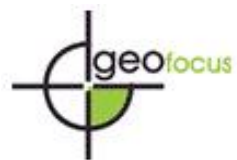

Garrido, J., Vilar, L., Echavarría, P., Martínez-Vega, J, Martín M. P. (2018): “¿Pueden las interfaces de usos del suelo explicar la ocurrencia de incendios forestales a escala provincial? Los casos de Zamora y Madrid", GeoFocus (Artículos), no 22, p. 71-95. ISSN: 1578-5157 http://dx.doi.org/10.21138/GF.611

$20^{\circ} \mathrm{C}$, y precipitaciones, según la zona, en torno a 400-1000 mm anuales (Comunidad de Madrid 2007, Agencia Estatal de Meteorología 2011, Nafría et al. 2013).

Las formaciones arbóreas que ocupan mayor extensión son los bosques perennifolios, presentes en las dos provincias, el matorral (Zamora), el matorral arbolado, los pastos y los bosques caducifolios (Madrid) (Comunidad de Madrid 2007, Castilla y León 2018, Diputación de Zamora 2018).

En este estudio se ha empleado el mapa de usos del suelo Climate Change InitiativeLand Cover (CCI-LC 2010, Defourny et al. 2017) con una resolución de 300 m. La Figura 2 muestra los porcentajes de los distintos usos del suelo en las provincias de estudio de acuerdo con la citada fuente.

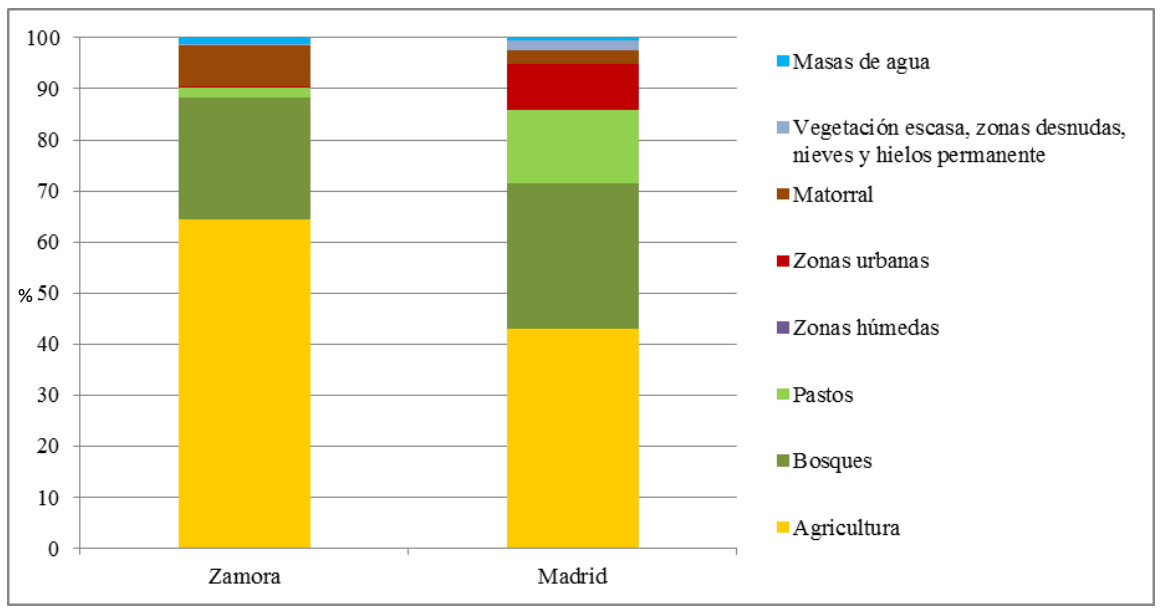

Figura 2. Porcentaje de categorías de uso del suelo según CCI-LC por provincia (2010).

Como se puede observar, en ambas provincias la agricultura ocupa el mayor porcentaje superficial, siendo este mayor en Zamora (64,5\%) que en Madrid ( 40\%). El uso urbano es muy importante en Madrid $(8,96 \%)$ y muy escaso en Zamora $(<1 \%)$. Los bosques ocupan en ambas provincias en torno al $25 \%$ de su superficie. Respecto a los pastos, en Zamora su presencia es escasa (inferior al $2 \%$ ) mientras que en Madrid ocupan un 14,47\%.

En el decenio 2001-2010 se produjeron unos 5.200 incendios en Zamora que afectaron a una superficie aproximada de 140.000 ha, mientras que, en Madrid, se registraron en torno a 1.000 siniestros que afectaron unas 25.000 ha.

La Figura 3 muestra el número total de siniestros (conatos (<1ha) e incendios) producidos entre los años 2005 y 2010 (Cubo et al. 2012). El año 2005 fue especialmente grave en lo que respecta a la ocurrencia de incendios en Zamora. En ese año se quemaron en la provincia unas 45.000 ha de superficie forestal. En 2006 se produjo un descenso en el número de siniestros y área quemada, volviendo a aumentar en los años sucesivos hasta 2009. En ambas provincias se observa un descenso importante de la incidencia de incendios en 2010. En Zamora el número de siniestros y superficie quemada es siempre superior en relación a Madrid. En el conjunto del periodo hubo 4.361 incendios en las dos provincias. Los incendios en Zamora fueron el $22 \%$ del total de los incendios de Castilla y León en este período 2005-2010 (3,2 \% del total de los de España) mientras que en Madrid se produjeron el 1,7 \% del total de España en el mismo período (Cubo et al. 2012). 
Garrido, J., Vilar, L., Echavarría, P., Martínez-Vega, J, Martín M. P. (2018): “¿Pueden las interfaces de usos del suelo explicar la ocurrencia de incendios forestales a escala provincial? Los casos de Zamora y Madrid", GeoFocus (Artículos), no 22, p. 71-95. ISSN: 1578-5157 http://dx.doi.org/10.21138/GF.611

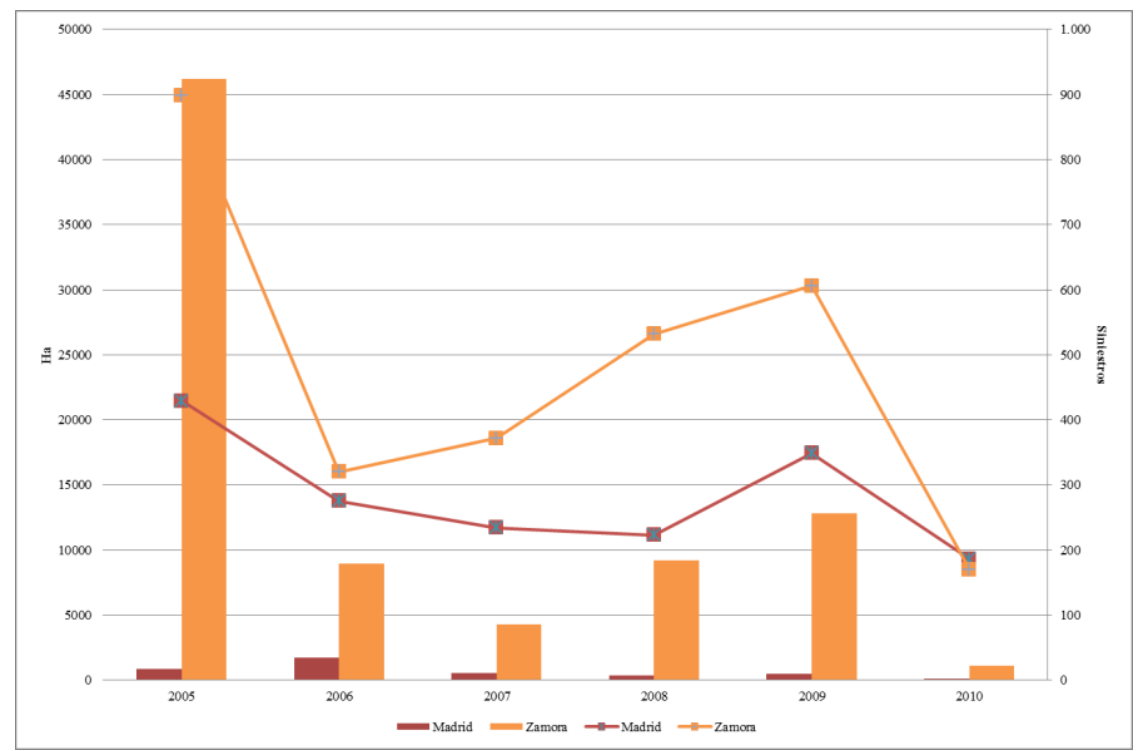

Figura 3. Evolución del número de siniestros (líneas) y superficie quemada (barras) en las provincias de estudio (2005-2010) (Cubo et al. 2012).

Respecto a la causalidad (Figura 4), las estadísticas indican que en la provincia de Zamora destaca el elevado porcentaje de intencionalidad (76,44 \%) mientras que en Madrid los incendios por causas desconocidas $(51,23 \%)$ y accidentes $(21,67 \%)$ son los más frecuentes (Cubo et al. 2012).

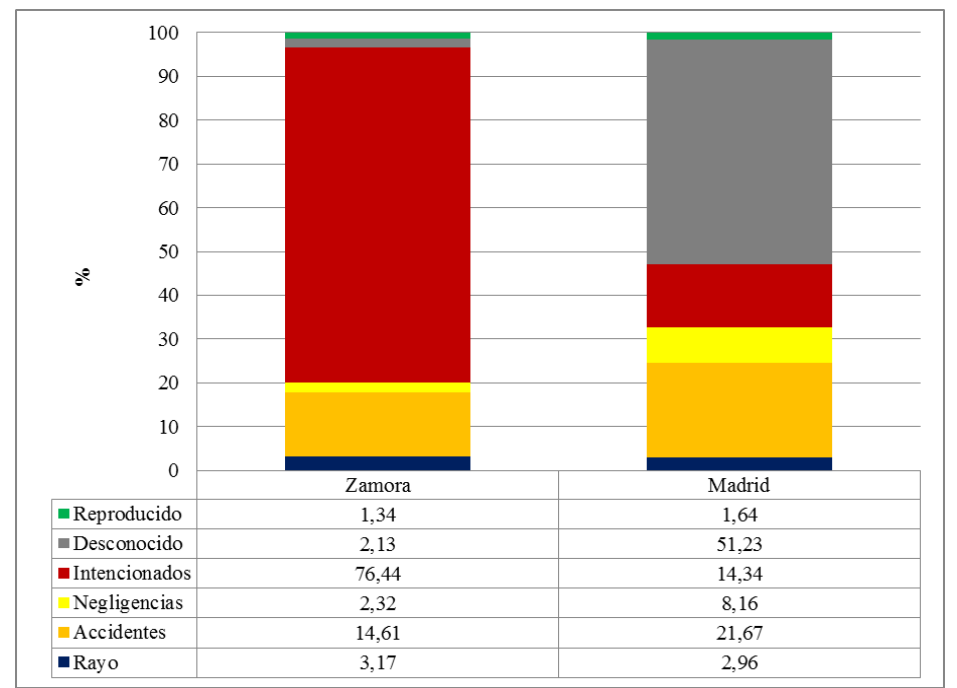

Figura 4. Número de siniestros por provincia y causa, 2001-2010) (Cubo et al. 2012).

2.2. Datos de entrada del modelo

Para llevar a cabo este estudio se han obtenido, por un lado, datos de ocurrencia histórica de incendios forestales (variable dependiente) y, por otro, interfaces de coberturas del suelo (variables independientes) de las dos provincias estudiadas referidas a una cuadrícula de $1 \mathrm{x} 1 \mathrm{~km}$, para el intervalo temporal comprendido entre los años 2005 y 2010. El período temporal elegido corresponde con la disponibilidad de datos de la variable dependiente y de los usos del suelo para la obtención de las interfaces en un periodo temporal coincidente; además, 


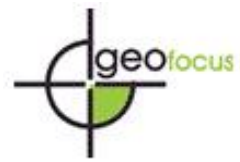

Garrido, J., Vilar, L., Echavarría, P., Martínez-Vega, J, Martín M. P. (2018): “¿Pueden las interfaces de usos del suelo explicar la ocurrencia de incendios forestales a escala provincial? Los casos de Zamora y Madrid", GeoFocus (Artículos), no 22, p. 71-95. ISSN: 1578-5157 http://dx.doi.org/10.21138/GF.611

se corresponde con un período interesante de cambio socio-económico (antes y después de la crisis de 2007) que ha tenido su reflejo en cambios de uso del suelo claves (urbanización e incendios forestales; Rodríguez-Rodríguez y Martínez-Vega 2017).

\subsubsection{Variable dependiente: puntos de ignición}

La variable dependiente (Figura 5) representa la ocurrencia de incendios en cada cuadrícula de referencia $1 \times 1 \mathrm{~km}$. Para su representación espacial se han utilizado los puntos de ignición del periodo 2005-2010, para la provincia de Madrid, y 2007-2010 para la de Zamora (Servicio de Incendios Forestales, Cuerpo de Bomberos, Comunidad de Madrid, Dirección General de Medio Natural, Junta de Castilla y León). Los incendios, con localización en coordenadas $(\mathrm{x}, \mathrm{y})$, fueron referidos a la cuadrícula de 1x1 km mediante la intersección espacial de ambas fuentes para el posterior cálculo de la presencia o ausencia de dichos puntos en cada cuadrícula.

a)

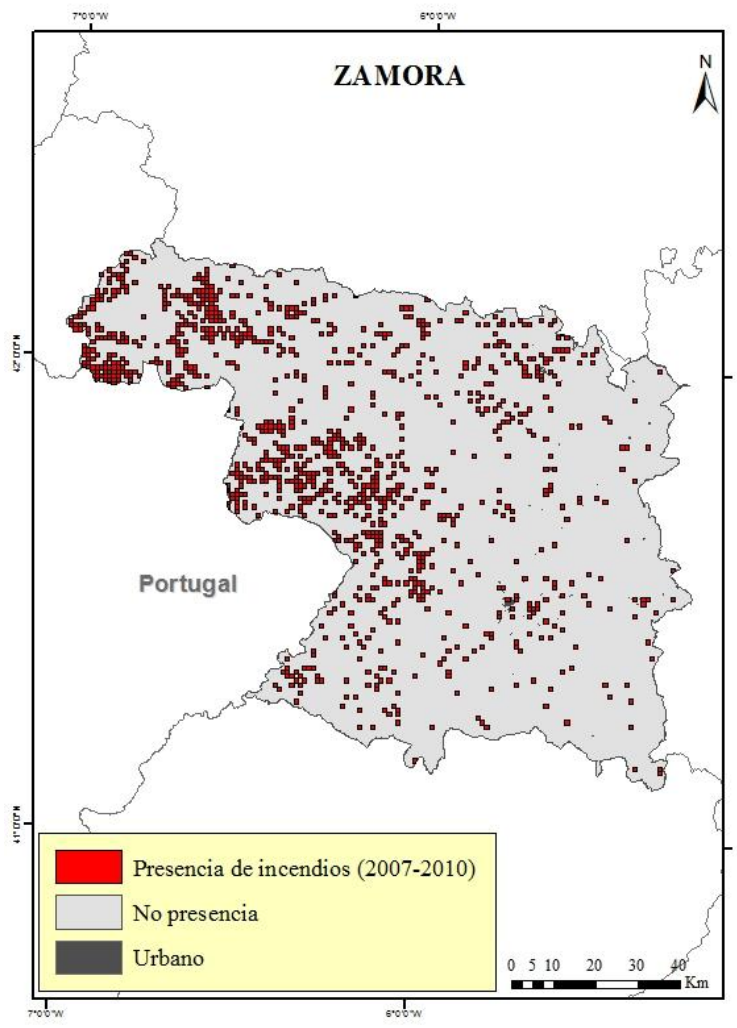

b)

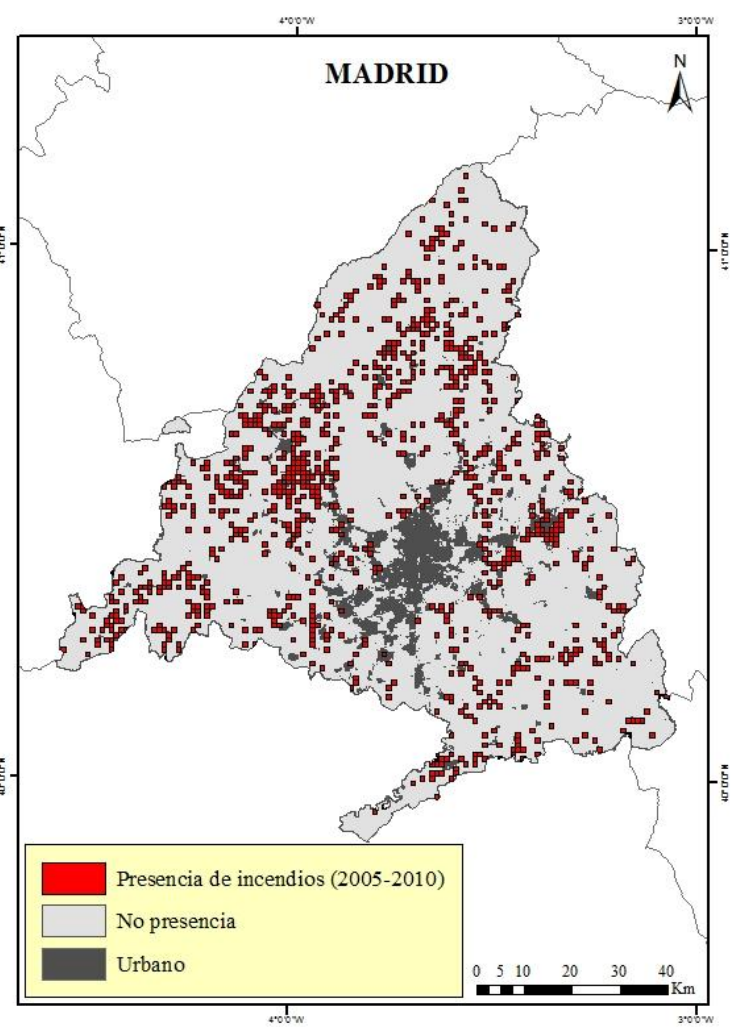

Figura 5. Variable dependiente: presencia de puntos de ignición de cuadrícula de 1x1 $\mathbf{k m}$. a) Zamora (2007-2010); b) Madrid (2005-2010).

\subsubsection{Variables independientes: Interfaces}

Las variables independientes empleadas en este estudio han sido las zonas de interfaz, definidas, de forma general, como las áreas de contacto entre los usos del suelo urbanos, agrícolas y pastos y los usos forestales. Como se ha mencionado anteriormente, en estas zonas de interfaz se desarrollan actividades humanas que pueden propiciar la ignición de incendios y 


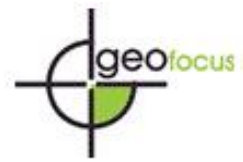

Garrido, J., Vilar, L., Echavarría, P., Martínez-Vega, J, Martín M. P. (2018): “¿Pueden las interfaces de usos del suelo explicar la ocurrencia de incendios forestales a escala provincial? Los casos de Zamora y Madrid", GeoFocus (Artículos), no 22, p. 71-95. ISSN: 1578-5157 http://dx.doi.org/10.21138/GF.611

han sido empleadas como variables predictoras en trabajos anteriores, en combinación con otras variables socio-económicas y ambientales (Leone et al. 2003, Vilar et al. 2008, Martínez et al. 2009, Vilar et al. 2011).

Para la elaboración de estas variables de interfaz se ha utilizado el mapa de usos del suelo CCI-LC de 2010. Este producto ha sido desarrollado por la ESA (European Space Agency, sus siglas en inglés) y proporciona coberturas de uso del suelo a escala global y a una resolución espacial de $300 \mathrm{~m}$, desde 1992 hasta 2015, con datos anuales, así como tres productos que engloban periodos de 5 años (1998-2002; 2003-2007, 2008-2012) (Defourny et al. 2017). Para este estudio la leyenda original del mapa CCI-LC, que incluye 37 categorías, ha sido reclasificada a 13 categorías (Tabla 1), utilizadas para el cálculo de las interfaces (Tabla 2). En esta segunda tabla se describen las 26 interfaces definidas para el estudio (7 urbanas, 13 agrícolas y 6 de pastos). En negrita se destacan las interfaces que engloban diversas subcategorías, a las que denominaremos generales, mientras que el resto corresponden a interfaces que engloban sólo algunos subtipos concretos dentro de las categorías generales urbano, agrícola, pasto y forestal. A estas últimas las denominaremos interfaces específicas. Aunque la interfaz podría limitarse espacialmente a la zona de contacto entre categorías de uso, dado que la resolución espacial del mapa de referencia es de $300 \mathrm{~m}$, la zona de interfaz corresponde, en este trabajo, a un buffer de dos píxeles, uno a cada lado de la zona de contacto. La espacialización de las zonas de interfaz a la unidad espacial de referencia del estudio se ha realizado mediante la intersección con la rejilla de $1 \times 1$ calculándose, a continuación, la proporción de área correspondiente a cada interfaz incluida en cada una de las cuadrículas (de 0 a $100 \%$ ). Los procesos de análisis espacial se han llevado a cabo con el programa ArcGIS 10.4 (ESRI 2014).

Tabla 1. Reclasificación de las categorías de uso del mapa CCI-LC 2010.

\begin{tabular}{|c|c|}
\hline CLASES RECLASIFICADAS & $\begin{array}{l}\text { CLASES ORIGINALES } \\
\text { CCI-LC } 2010\end{array}$ \\
\hline Forestal 1 (F1; Frondosas) & $50,60,61,62$ \\
\hline Forestal 2 (F2; Coníferas) & $70,71,72,80,81,82$ \\
\hline Forestal 3 (F3; Mixto) & 90 \\
\hline Forestal 4 (F4; Matorral) & $120,121,122$ \\
\hline $\begin{array}{c}\text { Forestal } 5 \text { (F5; Mosaico de arbolado y matorral con } \\
\text { herbáceas) }\end{array}$ & 100 \\
\hline Forestal 6 (F6; Pastos) & 110,130 \\
\hline Forestal total 1 (FT1, con Pastos) & $\mathrm{F} 1+\mathrm{F} 2+\mathrm{F} 3+\mathrm{F} 4+\mathrm{F} 5+\mathrm{F} 6$ \\
\hline Forestal total 2 (FT2, sin Pastos) & $\mathrm{F} 1+\mathrm{F} 2+\mathrm{F} 3+\mathrm{F} 4+\mathrm{F} 5$ \\
\hline $\begin{array}{l}\text { Agrícola } 1 \text { (A1; Cultivos herbáceos y arbóreos de secano, } \\
\text { cultivos de regadío, y mosaico de cultivos ( }>50 \% \text { ) con } \\
\text { vegetación natural (herbácea, arbustiva, arbórea, }<50 \%) \text { ) }\end{array}$ & $10,11,12,20,30$ \\
\hline $\begin{array}{c}\text { Agrícola } 2 \text { (A2; Mosaico de vegetación natural } \\
\text { (herbácea, arbustiva, arbórea, >50\%) con cultivos } \\
\qquad(<50 \%))\end{array}$ & 40 \\
\hline Agrícola total & $\mathrm{A} 1+\mathrm{A} 2$ \\
\hline Pastos & 110,130 \\
\hline Urbano & 190 \\
\hline
\end{tabular}


Garrido, J., Vilar, L., Echavarría, P., Martínez-Vega, J, Martín M. P. (2018): “¿Pueden las interfaces de usos del suelo explicar la ocurrencia de incendios forestales a escala provincial? Los casos de Zamora y Madrid", GeoFocus (Artículos), no 22, p. 71-95. ISSN: 1578-5157 http://dx.doi.org/10.21138/GF.611

Tabla 2. Interfaces IUF, IAF e IPF para las provincias de estudio. En sombreado, las interfaces para el Modelo interfaces generales.

\begin{tabular}{|c|c|c|}
\hline Interfaz & Clases CCI-LC & Descripción \\
\hline IUF Fron & Urbano $\|$ Forestal 1 & Urbano - Forestal Frondosas \\
\hline IUF Con & Urbano $\|$ Forestal 2 & Urbano - Forestal Coníferas \\
\hline IUF Mix & Urbano $\|$ Forestal 3 & Urbano - Forestal Mixto \\
\hline IUF Mat & Urbano $\|$ Forestal 4 & Urbano - Matorral \\
\hline IUF Mos & Urbano $\|$ Forestal 5 & Urbano - Mosaico de arbolado y matorral con herbáceas \\
\hline IUF Pas & Urbano $\|$ Forestal 6 & Urbano Pastos \\
\hline IUF & Urbano $\|$ Forestal total 1 & Urbano Forestal \\
\hline IAF CulFron & Agrícola 1 || Forestal 1 & Cultivos de secano/regadíos y mosaicos de cultivo - Forestal Frondosa \\
\hline IAF CulCon & Agrícola 1 || Forestal 2 & Cultivos de secano/regadíos y mosaicos de cultivo - Forestal Coníferas \\
\hline IAF CulMix & Agrícola 1 || Forestal 3 & Cultivos de secano/regadíos y mosaicos de cultivo - Forestal Mixto \\
\hline IAF CulMat & Agrícola 1 || Forestal 4 & Cultivos de secano/regadíos y mosaicos de cultivo - Matorral \\
\hline IAF CulMos & Agrícola 1 || Forestal 5 & $\begin{array}{l}\text { Cultivos de secano/regadíos y mosaicos de cultivo - Mosaico de arbolado y } \\
\text { matorral con herbáceas }\end{array}$ \\
\hline IAF CulPas & Agrícola 1 || Forestal 6 & Cultivos de secano/regadíos y mosaicos de cultivo - Pastos \\
\hline IAF MosFron & Agrícola 2 || Forestal 1 & Mosaico de vegetación natural y cultivos - Forestal Frondosas \\
\hline IAF MosCon & Agrícola 2 || Forestal 2 & Mosaico de vegetación natural y cultivos - Forestal Coníferas \\
\hline IAF MosMix & Agrícola 2 || Forestal 3 & Mosaico de vegetación natural y cultivos - Forestal Mixto \\
\hline IAF MosMat & Agrícola 2 || Forestal 4 & Mosaico de vegetación natural y cultivos - Matorral \\
\hline IAF MosMos & Agrícola 2 || Forestal 5 & $\begin{array}{c}\text { Mosaico de vegetación natural y cultivos - Mosaico de arbolado y matorral con } \\
\text { herbáceas }\end{array}$ \\
\hline IAF MosPas & Agrícola 2 || Forestal 6 & Mosaico de vegetación natural y cultivos - Pastos \\
\hline IAF & $\begin{array}{l}\text { Agrícola 1+Agrícola } 2 \| \\
\text { Forestal total } 1\end{array}$ & Agrícola - Forestal \\
\hline IPF Fron & Pastos || Forestal 1 & Pastos - Forestal Frondosas \\
\hline IPF Con & Pastos || Forestal 2 & Pastos - Forestal Coníferas \\
\hline IPF Mix & Pastos || Forestal 3 & Pastos- Forestal Mixto \\
\hline IPF Mat & Pastos \| Forestal 4 & Pastos - Matorral \\
\hline IPF Mos & Pastos || Forestal 5 & Pastos - Mosaico de arbolado y matorral con herbáceas \\
\hline IPF & Pastos $\|$ Forestal total 2 & Pastos - Forestal (sin pastos) \\
\hline
\end{tabular}

La categoría urbana se refiere a la clase asentamientos (190) del mapa CCI-LC que no está desglosada en subcategorías (i.e. urbano compacto y urbanizaciones ajardinadas) por lo que todas las interfaces que incluyen la categoría "urbano" se refieren a una única categoría global. Las categorías agrícolas $(10,11,12,20,30,40)$ de la leyenda original se han reclasificado en dos: A1, categorías más puramente agrícolas $(10,11,12,20$ y 30), que se han utilizado para definir la IAF Cul y A2, que engloba usos que pueden considerarse de transición entre zonas agrícolas y forestales (40) y definirían la IAF Mos. Esta última, se ha desglosado para valorar si podrían considerarse zonas relacionadas con el abandono de tierras cultivadas donde las causas de ignición son distintas que en las tierras de cultivo. Las clases incluidas en la IPF del CCI-LC son los mosaicos de cobertura herbácea $(>50 \%)$ con árboles y matorral $(<50 \%)$ y los pastos 


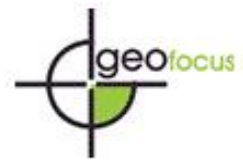

Garrido, J., Vilar, L., Echavarría, P., Martínez-Vega, J, Martín M. P. (2018): “¿Pueden las interfaces de usos del suelo explicar la ocurrencia de incendios forestales a escala provincial? Los casos de Zamora y Madrid", GeoFocus (Artículos), no 22, p. 71-95. ISSN: 1578-5157 http://dx.doi.org/10.21138/GF.611

propiamente dichos (clases 110 y 130 de la leyenda original), excluidas de las categorías forestales para poder obtener una categoría propia de pastos que no fuese redundante con la forestal. Las cinco categorías de la interfaz pasto-forestal se han elaborado a partir del contacto de las formaciones vegetales (F1-F5) con las coberturas de pastos, excluyendo éstos obviamente. Las distintas coberturas forestales se han reclasificado en seis categorías para observar las diferentes relaciones que tenía cada tipo de interfaz -forestal (coníferas, bosque mixto, caducifolias...), urbano y agrícola- con la ignición.

La Figura 6 muestra la distribución espacial de las tres interfaces generales en las dos provincias de estudio. La IUF en Madrid $\left(1.023 \mathrm{~km}^{2}\right)$ ocupa mayor extensión que en Zamora $\left(8,6 \mathrm{~km}^{2}\right)$. Se evidencia la asociación de IUF con la red radioconcéntrica de las carreteras madrileñas. La IAF presenta una extensión similar en las dos provincias: $2.684 \mathrm{~km}^{2}$ en Zamora y $2.761 \mathrm{~km}^{2}$ en Madrid. La IPF tiene mayor extensión en Madrid $\left(1.023 \mathrm{~km}^{2}\right)$ que en Zamora $\left(534 \mathrm{~km}^{2}\right)$. Se ubican mayoritariamente en la mitad occidental de ambas.
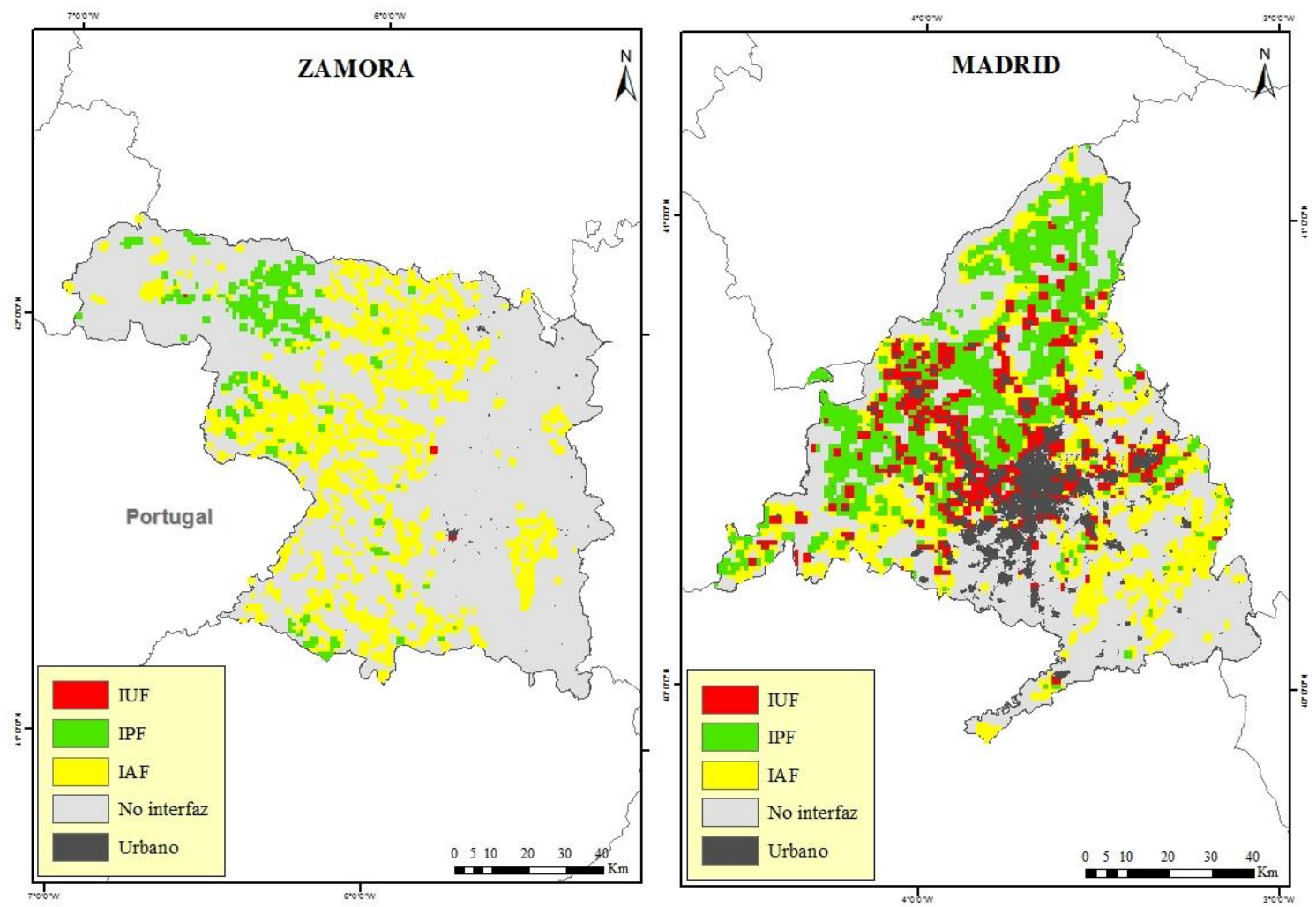

Figura 6. Interfaces totales del modelo de interfaces generales (IAF, IPF e IUF) referenciadas a la rejilla de 1x1 km en las provincias de estudio. En cada celda se representa la presencia de cada interfaz superpuesta (IUF, IPF, IAF).

En la Figura 7 se muestran las 18 interfaces específicas más representativas en Zamora y Madrid empleadas en el modelo de interfaces específicas. En Zamora, las interfaces específicas derivadas de la IUF no tienen gran representación, no así en Madrid, donde destaca la IUF Pas, que ocupa la mayor extensión (44\% de la IUF). En las interfaces específicas derivadas de la IPF en Zamora destacan la IPF Mos y la IPF Mat ( 40\% de la IPF). En Madrid, la mayor extensión pertenece a la IPF Fron (75 \% de la IPF). Las IAF con mayor presencia en 


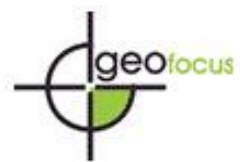

Garrido, J., Vilar, L., Echavarría, P., Martínez-Vega, J, Martín M. P. (2018): “¿Pueden las interfaces de usos del suelo explicar la ocurrencia de incendios forestales a escala provincial? Los casos de Zamora y Madrid", GeoFocus (Artículos), no 22, p. 71-95. ISSN: 1578-5157 http://dx.doi.org/10.21138/GF.611

Zamora son la IAF MosMos e IAF MosMat (80 \% de la IAF). En Madrid, la IAFCul Pas y la IAF CulFron ocupan el $~ 50 \%$ de la IAF.a)
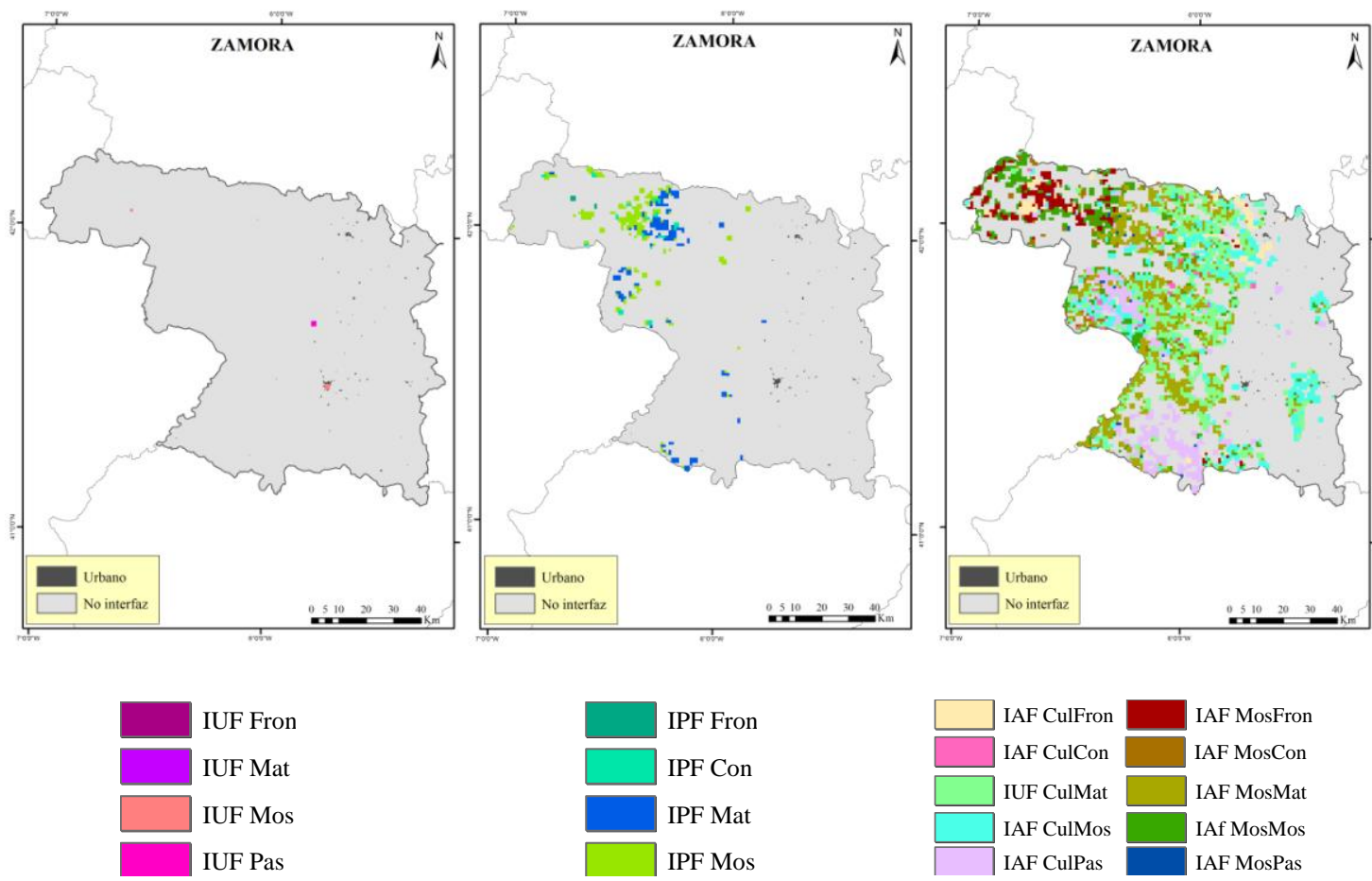

b)
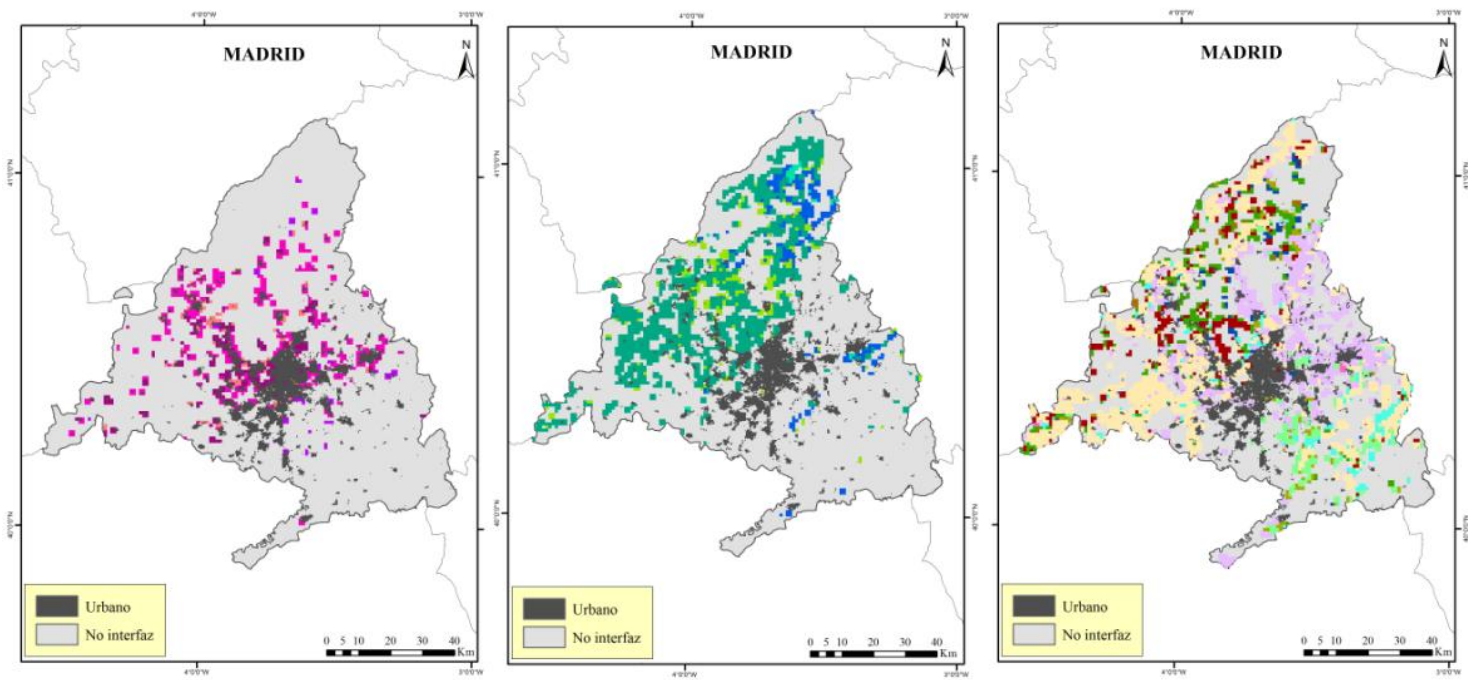

Figura 7. Interfaces desglosadas del modelo de interfaces específicas referenciadas a la rejilla de 1x1 km en las provincias de estudio: a) Zamora; b) Madrid. En cada celda se representa la presencia de cada interfaz desglosada superpuesta (IUF, IPF, IAF). El significado de las abreviaturas de las interfaces se puede consultar en la Tabla 2. 
Garrido, J., Vilar, L., Echavarría, P., Martínez-Vega, J, Martín M. P. (2018): “¿Pueden las interfaces de usos del suelo explicar la ocurrencia de incendios forestales a escala provincial? Los casos de Zamora y Madrid", GeoFocus (Artículos), no 22, p. 71-95. ISSN: 1578-5157 http://dx.doi.org/10.21138/GF.611

\subsection{Modelización de la ocurrencia de incendio}

Los modelos de probabilidad de ocurrencia de incendios forestales se realizaron empleando MaxEnt 3.3.3k (Phillips et al. 2006). MaxEnt está creado y diseñado para utilizar datos de presencia que, en este estudio, se corresponden con las celdas de $1 \mathrm{~km}^{2}$ en las que se ha localizado uno o más puntos de ignición en el período analizado. En este caso, las celdas donde no se registrado ningún incendio (valor 0 de la variable dependiente) no se interpretan como "ausencia" de incendios por el modelo, ya que estas zonas son susceptibles de sufrir un incendio forestal si comparten las características ambientales y socio-económicas de las zonas propensas a padecerlos (Parisien et al. 2012). Para cada celda con incendio, MaxEnt calcula la probabilidad de ocurrencia ajustando la distribución de probabilidad de entropía máxima (la más uniforme) a las variables independientes (Phillips et al. 2006, Deblauwe et al. 2008, Parisien et al. 2012). El algoritmo de MaxEnt evalúa, iterativamente, los contrastes entre las celdas con incendio y el resto y, además, tiene la capacidad de ajustar y adaptar relaciones no lineales entre la variable dependiente y las variables independientes, de tal manera que los modelos pueden describir relaciones complejas (Parisien y Moritz 2009, Parisien et al. 2012). El resultado de MaxEnt es una estimación relativa, siendo el valor de cada una de las celdas del mapa resultante una estimación de la probabilidad de presencia de incendio. MaxEnt suministra una serie de modelos de diagnóstico. Uno de ellos, el que ha sido utilizado en este estudio, es el de la contribución relativa de cada variable al modelo, que, además, considera las interacciones entre las distintas variables mediante su tipo de función (Parisien y Moritz 2009) y permite dotar de un cierto componente explicativo al modelo.

En este estudio se han obtenido con MaxEnt dos modelos para cada provincia: uno utilizando como variables independientes las interfaces generales y otro usando las interfaces específicas (Tabla 2). Para la elaboración de los modelos se han utilizado el $75 \%$ de las celdas con presencia de incendio para la calibración. El $25 \%$ restante y 1600 celdas aleatorias sin incendio se han empleado para la validación. Además, excluyendo una variable por vez, el test de MaxEnt jack-knife evaluó el porcentaje de la contribución relativa de cada variable para el ajuste del modelo (Baldwin 2009, Phillips et al. 2009, Elith et al. 2010). El ajuste de la predicción de los modelos obtenidos con MaxEnt ha sido evaluado a través de la curva operada por el receptor (ROC, por sus siglas en inglés, Receiver Operating Characteristic; Egan (1975)). El área bajo la curva ajustada (AUC, por sus siglas en inglés, Area Under the Curve) indica la probabilidad de que, ante un par de casos (presencia y ausencia de incendio), el modelo los clasifique correctamente. El rango de AUC está entre 0 y $1:<0,5$ significa que no hay discriminación; 0,5-0,69 débil; 0,7-0,79 razonable; 0,8-0,89 buena; y 0,9-1 excelente (Hanley y McNeil 1982). Además, a partir de tabulaciones cruzadas de las clasificaciones de los modelos se han proporcionado los porcentajes de idoneidad (verdaderos positivos o presencias en áreas predichas como favorables para que se produzca un incendio) y el error de omisión (falsos negativos o presencia de incendios en zonas predichas como no favorables (Vilar et al. 2016).

Se llevó a cabo, además, una validación independiente de los resultados con datos de incendios correspondientes al año 2011. Para ello fueron superpuestos los puntos de ignición del año 2011 para observar en qué zonas de los modelos se habían producido y, así, comprobar la eficacia de los modelos general y específico en Zamora y Madrid. Se llevaron a cabo intersecciones de estos incendios con los mapas de probabilidad obtenidos clasificando los valores de probabilidad en cinco categorías de 0 a 1 (Muy baja: >0-0,2; Baja: 0,2-0,4; Media: 0,4-0,6; Alta: 0,6-0,8; Muy alta: 0,8-1) para así comprobar en qué categorías de probabilidad estimada se habían producido los incendios del año 2011. 


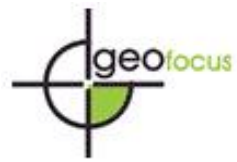

Revista Internacional de Ciencia y Tecnología de la Información Geográfica International Review of Geographical Information Science and Technology

Garrido, J., Vilar, L., Echavarría, P., Martínez-Vega, J, Martín M. P. (2018): “¿Pueden las interfaces de usos del suelo explicar la ocurrencia de incendios forestales a escala provincial? Los casos de Zamora y Madrid", GeoFocus (Artículos), no 22, p. 71-95. ISSN: 1578-5157 http://dx.doi.org/10.21138/GF.611

\section{Resultados}

En la Figura 8 se observa la distribución espacial de las probabilidades de ocurrencia de incendio obtenidas para cada modelo y provincia.

a)
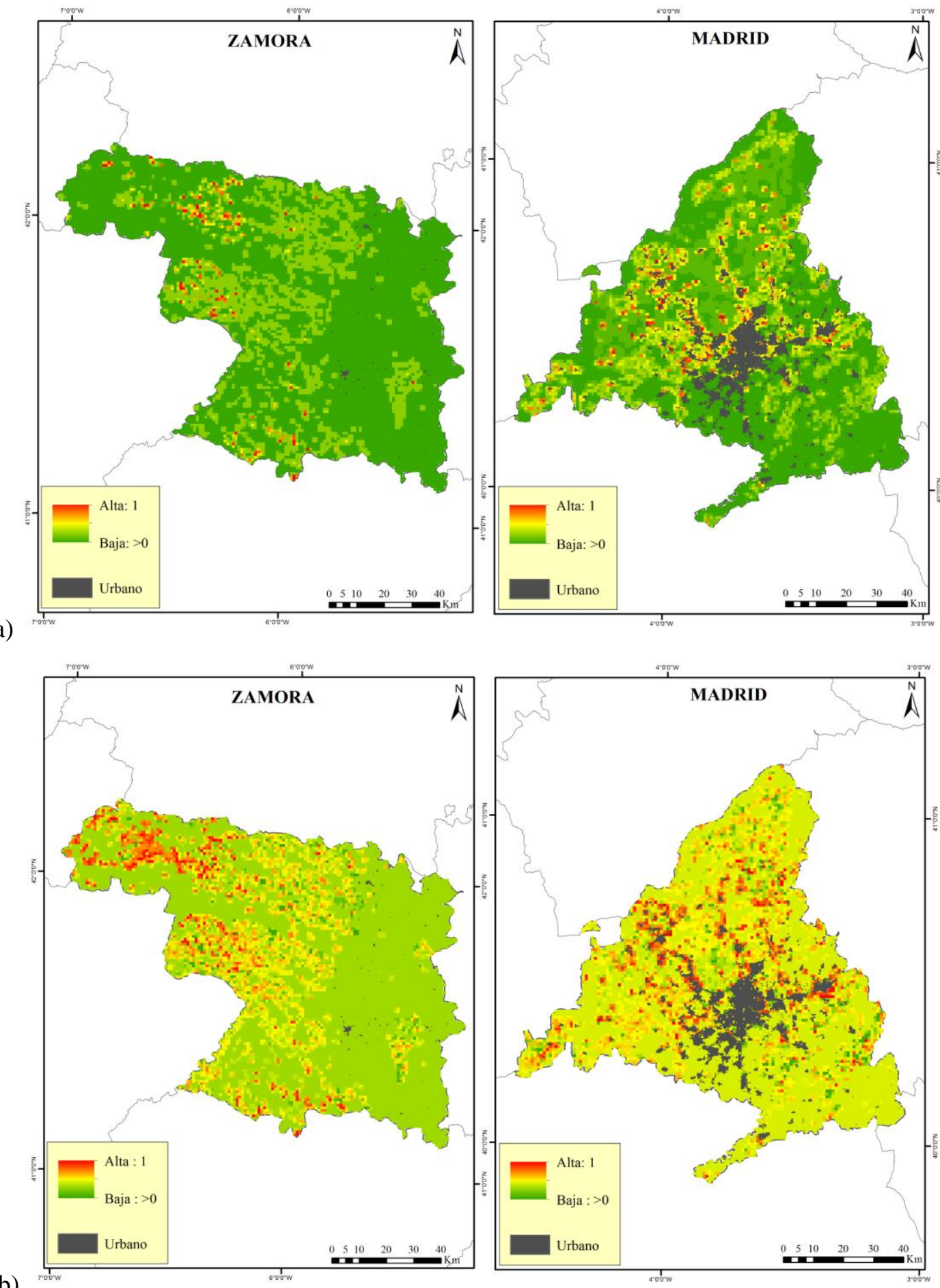

b)

Figura 8. Mapas de probabilidad de ocurrencia de incendio obtenida con MaxEnt: a) interfaces generales; b) interfaces específicas. 


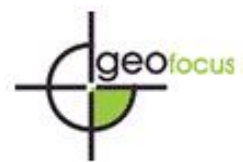

Garrido, J., Vilar, L., Echavarría, P., Martínez-Vega, J, Martín M. P. (2018): “¿Pueden las interfaces de usos del suelo explicar la ocurrencia de incendios forestales a escala provincial? Los casos de Zamora y Madrid", GeoFocus (Artículos), no 22, p. 71-95. ISSN: 1578-5157 http://dx.doi.org/10.21138/GF.611

Los resultados de los modelos basados en interfaces generales (Figura 8 a) muestran que en Zamora las zonas con valores más altos de probabilidad de ocurrencia se ubican en el noroeste, oeste y sur. En Madrid se localizaron en el suroeste, oeste, noroeste, norte y este. En Madrid estas áreas con alta probabilidad de ocurrencia, de forma general, coinciden con los ejes viarios y las zonas de interfaz urbano-forestal (Figura 6). En Zamora la alta probabilidad coincide, a grandes rasgos, con las zonas de interfaz pasto-forestal.

En cuanto a los modelos basados en interfaces específicas (Figura 8 b) la distribución espacial de la probabilidad de ocurrencia de incendio en ambas provincias es muy similar a la del modelo de interfaces generales, salvo en el sector noroeste de Zamora donde las probabilidades altas han ocupado mayor superficie. La diferencia fundamental entre los dos modelos (general y específico) es que el segundo muestra probabilidades de ocurrencia más altas en todo el territorio, con un aumento general de las probabilidades medias y una intensificación de los valores altos.

La Tabla 3 muestra las métricas de evaluación de ajuste obtenidas para los modelos general y específico.

Tabla 3. Verdaderos positivos, falsos negativos y Area Under the Curve (AUC) de los modelos general y específico para cada provincia. En negrita, los valores más altos.

\begin{tabular}{ccc}
\hline & Zamora & \\
\hline & $\begin{array}{c}\text { Modelo de interfaces } \\
\text { Generales }\end{array}$ & $\begin{array}{c}\text { Modelo de interfaces } \\
\text { Específicas }\end{array}$ \\
Verdaderos positivos (\%) & 29,7 & $\mathbf{3 0 , 8}$ \\
Falsos negativos (\%) & 56 & $\mathbf{4 9}$ \\
AUC & 0,55 & $\mathbf{0 , 6 5}$ \\
\hline & Madrid & \\
& Modelo de interfaces & $\begin{array}{c}\text { Modelo de interfaces } \\
\text { Específicas }\end{array}$ \\
Verdaderos positivos (\%) & $\mathbf{5 9}$ & 47,4 \\
Falsos negativos (\%) & $\mathbf{0}$ & 17,3 \\
AUC & 0,6 & $\mathbf{0 , 6 9}$ \\
\hline
\end{tabular}

Los ajustes generales medidos por AUC fueron mejores en los modelos de interfaces específicas en ambas provincias, pasando de 0,55 a 0,65 en Zamora y de 0,6 a 0,69 en Madrid. Respecto a los aciertos en la identificación de verdaderos positivos, los modelos generales ofrecieron mejores resultados que los específicos en Madrid (59\% frente a 47,4 \%), mientras que, en Zamora, fue ligeramente mejor el modelo específico (30,8\% frente a 29,7\%). Por lo que respecta a los falsos negativos, se observa la misma tendencia que en los verdaderos positivos. Estos resultados indican que, en el caso de Zamora, el $56 \%$ de los incendios ocurridos se clasificaron en zonas no favorables según el modelo general, disminuyendo al $49 \%$ en el modelo específico. Puede decirse entonces que, en Madrid, el modelo general ha proporcionado mejores resultados con un porcentaje mayor de verdaderos positivos y menor de falsos negativos que el modelo específico. En Zamora, en cambio, se observa el caso contrario, el modelo específico predice mejor la ocurrencia que el general. 
Garrido, J., Vilar, L., Echavarría, P., Martínez-Vega, J, Martín M. P. (2018): “¿Pueden las interfaces de usos del suelo explicar la ocurrencia de incendios forestales a escala provincial? Los casos de Zamora y Madrid", GeoFocus (Artículos), no 22, p. 71-95. ISSN: 1578-5157 http://dx.doi.org/10.21138/GF.611

La Tabla 4 muestra la relación de variables (interfaces) que más han contribuido a cada modelo en cada una de las provincias. En Zamora las variables que más aportaron fueron la IAF $(62,7 \%)$, en el modelo general, y la IAF MosFron (29,9\%), en el modelo específico, seguidas de IAF MosMos (26,2 \%) y IAF CulPas (19,5\%). Es decir, la interfaz agrícola-forestal es la que más contribuye a explicar la ocurrencia en el modelo general mientras que, en el modelo específico, de las posibles combinaciones de usos en esta interfaz, las que más influencia tienen son las zonas de mosaico agrícola con vegetación natural en contacto con bosques de frondosas y con zonas de mosaico de arbolado y matorral con herbáceas, así como entre zonas de cultivo y zonas de pastizal.

En Madrid fueron la IUF (64,6\%), en el modelo general, y la IUF Pas $(38,1 \%)$, seguida de IAF CulFron (10,4\%) e IAF CulPas $(9,4 \%)$ en el específico las que contribuyeron en mayor porcentaje. Aquí observamos que la interfaz específica que más influye es la que representa zonas de contacto entre áreas urbanas y de pastizal. El modelo específico permite también observar que las zonas de contacto agrícola con bosques de frondosas y pastizales contribuyen también, aunque en menor medida.

Tabla 4. Contribución (\%) de las interfaces más representativas de los modelos general y específico por provincias. En sombreado, los porcentajes más altos de cada modelo y provincia.

\begin{tabular}{|c|c|c|c|}
\hline & Variables & $\begin{array}{c}\text { Zamora } \\
(\%)\end{array}$ & $\begin{array}{c}\text { Madrid } \\
(\%)\end{array}$ \\
\hline \multirow{3}{*}{$\begin{array}{l}\text { Modelo } \\
\text { general }\end{array}$} & IUF & 0,2 & 64,6 \\
\hline & IAF & 62,7 & 23,9 \\
\hline & IPF & 37,1 & 11,5 \\
\hline \multirow{12}{*}{$\begin{array}{c}\text { Modelo } \\
\text { específico }\end{array}$} & IUF Fron & - & 4,3 \\
\hline & IUF Mat & - & 0,9 \\
\hline & IUF Mos & - & 2,3 \\
\hline & IUF Pas & - & 38,1 \\
\hline & IAF CulFron & 0,2 & 10,4 \\
\hline & IAF CulCon & 1,3 & 0,3 \\
\hline & IAF CulMat & 3,9 & 0,2 \\
\hline & IAF CulMos & 3 & 3,4 \\
\hline & IAF CulPas & 19,5 & 9,4 \\
\hline & IAF MosFron & 29,9 & 0,8 \\
\hline & IAF MosCon & 0,9 & 0,2 \\
\hline & IAF MosMat & 3,8 & 6,5 \\
\hline
\end{tabular}




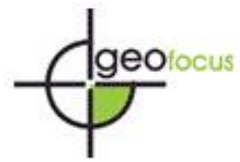

Garrido, J., Vilar, L., Echavarría, P., Martínez-Vega, J, Martín M. P. (2018): “¿Pueden las interfaces de usos del suelo explicar la ocurrencia de incendios forestales a escala provincial? Los casos de Zamora y Madrid", GeoFocus (Artículos), no 22, p. 71-95. ISSN: 1578-5157 http://dx.doi.org/10.21138/GF.611

\begin{tabular}{ccc}
\hline IAF MosMos & 26,2 & 6,5 \\
IAF MosPas & 2,6 & 2 \\
\hline IPF Fron & - & 2,6 \\
IPF Con & 0,2 & 0,1 \\
IPF Mat & 5,1 & 4,5 \\
IPF Mos & 3,5 & 7,5 \\
\hline
\end{tabular}

Los resultados de la validación independiente llevada a cabo con los puntos de ignición del año 2011 se muestran en la Figura 9. En el modelo general, más del $80 \%$ de los incendios del año 2011 sucedieron en zonas clasificadas de probabilidad media en ambas provincias. En cambio, en el modelo específico, el $\sim 40 \%$ de los incendios se iniciaron en zonas de probabilidad baja.

a)
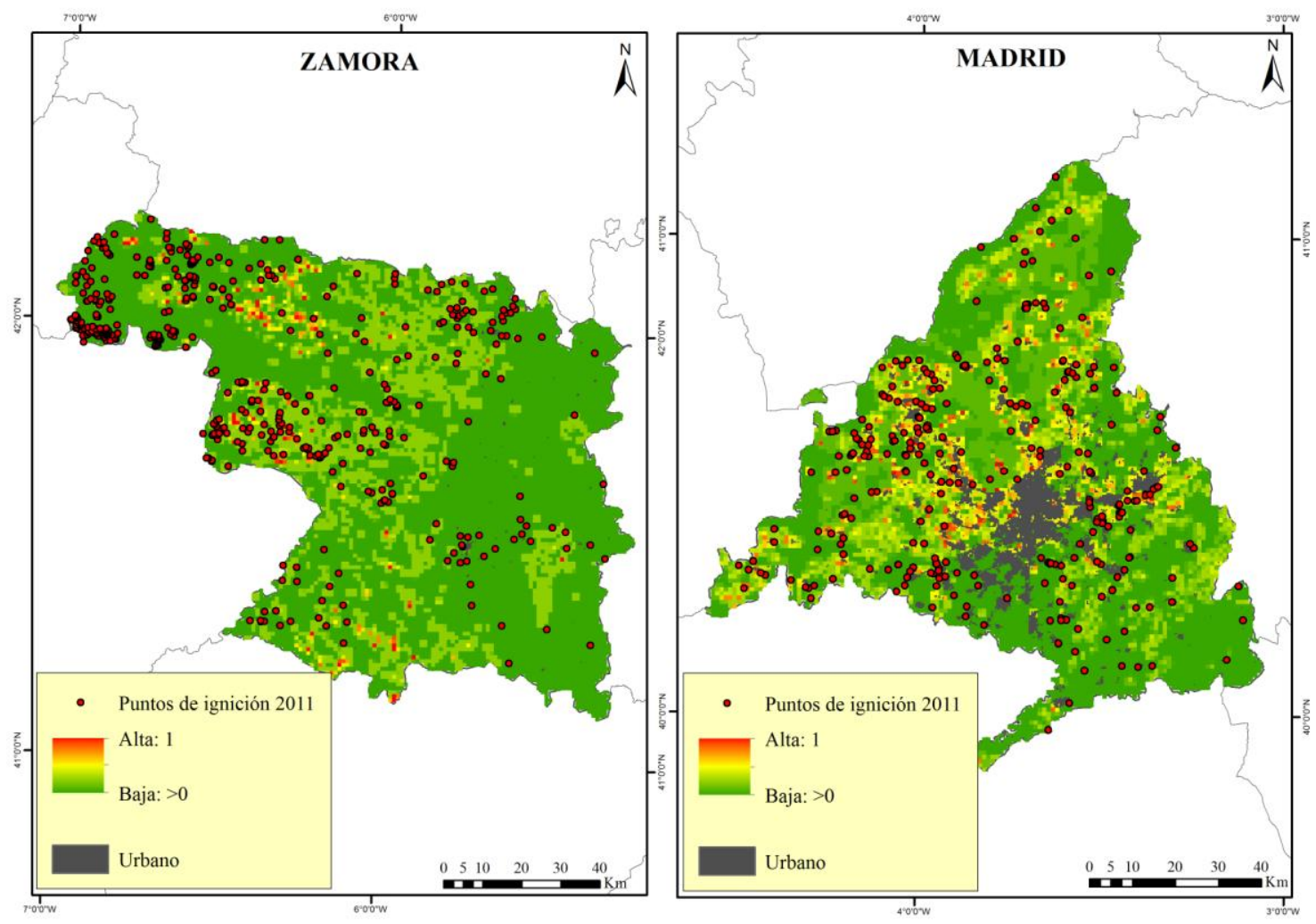


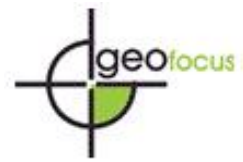

Garrido, J., Vilar, L., Echavarría, P., Martínez-Vega, J, Martín M. P. (2018): “¿Pueden las interfaces de usos del suelo explicar la ocurrencia de incendios forestales a escala provincial? Los casos de Zamora y Madrid", GeoFocus (Artículos), no 22, p. 71-95. ISSN: 1578-5157 http://dx.doi.org/10.21138/GF.611

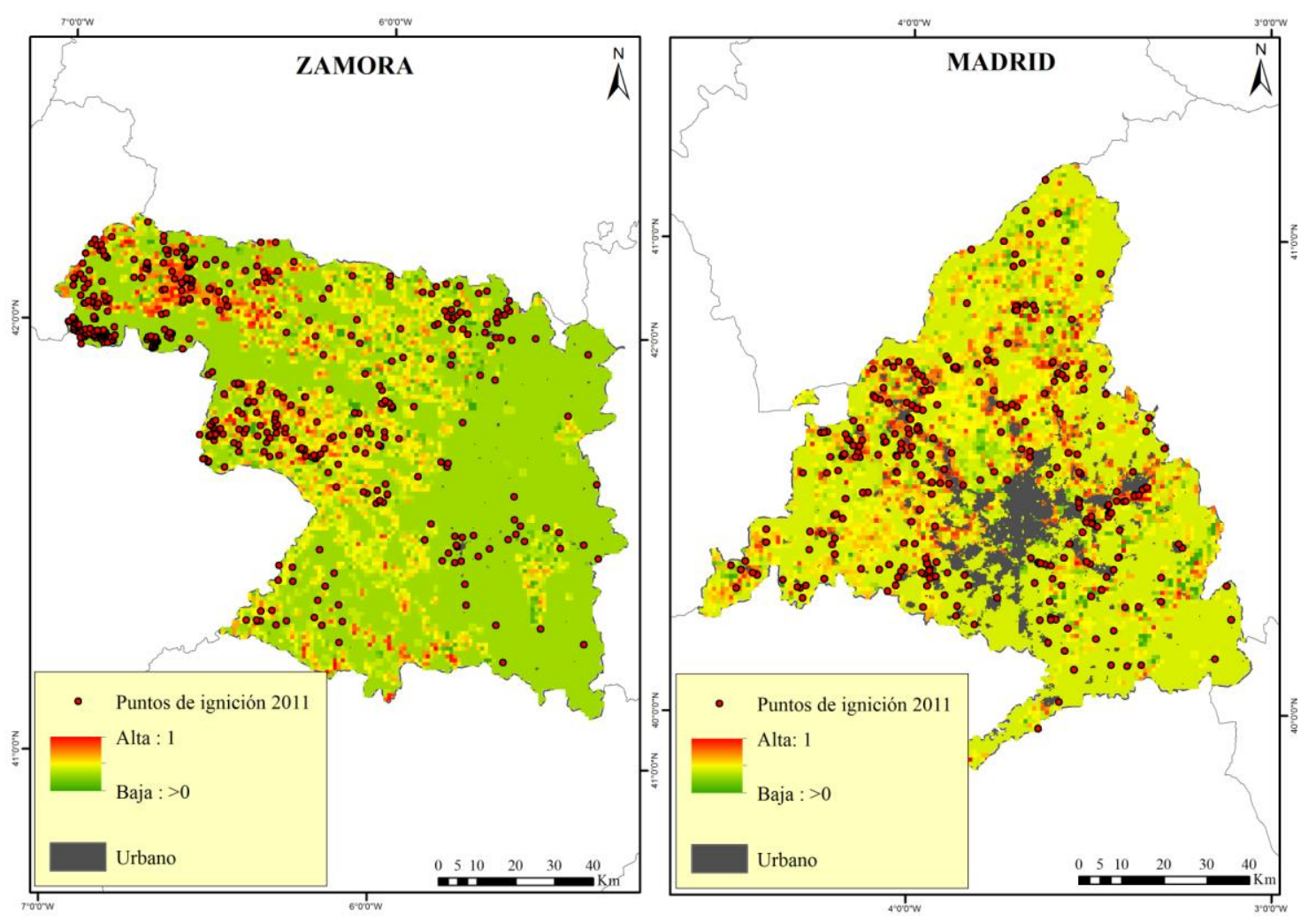

Figura 9. Validación independiente. Puntos de ignición de 2011 de las provincias de Zamora y Madrid superpuestos al modelo general (a) y específico (b).

\section{Discusión}

Trabajos anteriores han utilizado las interfaces como variables explicativas/predictivas de la ocurrencia de incendios forestales (Chou 1993, Syphard et al. 2007, Padilla y Vega-García 2011), pero no constan precedentes del uso, como en este trabajo, de interfaces específicas que descienden al nivel de formaciones vegetales permitiendo una mayor precisión en la predicción espacial de la ocurrencia.

Se han analizado dos provincias españolas con características muy diferentes en cuanto a usos del suelo relacionados con unas condiciones ambientales y, sobre todo, socio-económicas muy distintas. Estas diferencias se traducen en patrones distintos de ocurrencia y causalidad de incendios forestales, lo que nos ha permitido comprobar la validez de esta aproximación metodológica propuesta en casos de estudio discordes. Los resultados indican que las interfaces permiten explicar una parte importante de la ocurrencia en ambas provincias y que existen diferencias entre ellas en los que respecta a las interfaces que más influyen en el modelo. En Madrid, estas interfaces están claramente relacionadas con los usos urbanos y su contacto con zonas forestales donde habitualmente se producen incendios o conatos relacionados con acciones negligentes o accidentales en el uso del fuego (Vilar et al. 2010). En Zamora, en cambio, la ocurrencia de incendios se explica fundamentalmente por la presencia de zonas de contacto entre áreas de pasto y forestales, lo que podría estar relacionado con un uso negligente 
Garrido, J., Vilar, L., Echavarría, P., Martínez-Vega, J, Martín M. P. (2018): “¿Pueden las interfaces de usos del suelo explicar la ocurrencia de incendios forestales a escala provincial? Los casos de Zamora y Madrid", GeoFocus (Artículos), no 22, p. 71-95. ISSN: 1578-5157 http://dx.doi.org/10.21138/GF.611

del fuego en actividades tradicionales en entornos agrícolas y ganaderos que desembocan en la propagación de incendios a zonas forestales (Rodrigues et al. 2014, Vilar et al. 2016).

Los modelos basados en interfaces han conseguido un ajuste débil en las dos provincias (AUC entre 0,5 y 0,6), debido a la falta de otras variables explicativas de la ocurrencia de incendios, como las que representan factores biofísicos (topografía, clima, etc.). Sin embargo, permiten identificar factores de riesgo asociados a los usos del suelo que pueden obtenerse y actualizarse fácilmente $\mathrm{y}$, por tanto, pueden incorporarse de manera sencilla a los sistemas regionales de gestión de incendios. El empleo de interfaces específicas ha permitido concretar la localización espacial del riesgo al referirse a zonas de contacto de formaciones vegetales concretas. No obstante, el uso de interfaces específicas ha dado lugar a un aumento de los errores de omisión (falsos negativos) y un ligero descenso de los verdaderos positivos en Madrid. Esto puede ser debido a que la variable con mayor contribución en los modelos fue la IUF total en el modelo general y una de las IUF desglosadas (IUF Pas) en el modelo específico, repartiendo, por tanto, su contribución en este modelo con variables relacionadas con la IAF. Al desglosarse, la variable IUF total ha perdido número de píxeles e importancia para la localización aleatoria de los puntos necesarios para el background, lo que ha podido provocar un incremento en los errores finales de idoneidad y omisión. Sin embargo, en el caso de Zamora, el desglose de interfaces aumentó ligeramente los verdaderos positivos y disminuyó los falsos negativos, mejorando el ajuste global del modelo. En esta provincia, las interfaces específicas que más han contribuido al modelo son las de tipo agrícola, al igual que en el modelo general, por lo que la contribución se ha repartido entre interfaces del mismo tipo, aunque más detalladas.

En relación con la capacidad explicativa de las distintas interfaces, la IAF contribuyó un $62,7 \%$ en el modelo general de Zamora y un 23,9\% en el de Madrid. Estos resultados coincidieron con los de Rodrigues et al. (2014), quienes han demostrado, aplicando el modelo de regresión de GWLR, que esta interfaz contribuye, en gran modo, a la ocurrencia de incendios en su modelo para el conjunto de España (mayor coeficiente de regresión que las variables de accesibilidad - pistas forestales- o que la densidad de población -IUF-), pero con un valor más bajo en Madrid. Las categorías desglosadas de IAF son las que más han contribuido al modelo específico de Zamora, correspondiendo con zonas de mosaicos de arbolado y matorral con herbáceas. En este análisis, la IUF ha tenido una contribución relevante en Madrid, lo que coincide con los resultados obtenidos por Padilla y Vega-García (2011), que elaboraron un modelo de regresión logística para España en el período de 2002 a 2005; y Vilar et al. (2016), que obtuvieron un modelo multitemporal para la Comunidad de Madrid comparando GLM y MaxEnt que incluía, entre los factores socio-económicos relacionados con la ocurrencia de incendios, las interfaces IUF, IAF e IPF. La IUF también ha presentado una contribución notable a la ocurrencia de incendios forestales en Madrid en el trabajo de Rodrigues et al. (2014), en el período 1988-2011, y en Chuvieco et al. (2010), durante el período de 1990-2004. Los resultados de la IPF en Madrid son similares a los del trabajo de Vilar et al. (2016), donde la contribución a la ocurrencia de incendios ha disminuido con el tiempo (1980s-2000s), tanto en el modelo GLM como en MaxEnt. Es interesante ver que, en el modelo específico realizado en Madrid, aparece una contribución pequeña pero significativa (en torno al $10 \%$ ) de interfaces relacionadas con los usos agrícolas que, aunque residuales, parecen tener también su contribución al riesgo de incendios en la región.

Con relación a la distribución espacial de la probabilidad estimada de la ocurrencia de incendios, los patrones espaciales han sido muy similares en los modelos generales y específicos 
Garrido, J., Vilar, L., Echavarría, P., Martínez-Vega, J, Martín M. P. (2018): “¿Pueden las interfaces de usos del suelo explicar la ocurrencia de incendios forestales a escala provincial? Los casos de Zamora y Madrid", GeoFocus (Artículos), no 22, p. 71-95. ISSN: 1578-5157 http://dx.doi.org/10.21138/GF.611

en las dos provincias. Sin embargo, los valores de probabilidad se acentúan en los modelos específicos, es decir, en zonas donde los modelos generales predicen valores bajos pasan a medios y los valores altos se mantienen. El empleo de interfaces más específicas ha dado lugar, por tanto, a incrementos en los valores de la probabilidad, lo que implicaría unas medidas de prevención de incendios más proactivas en una parte mayor del territorio estudiado. En MaxEnt, al ser un modelo de sólo presencia (en este caso, incendios), no se tiene el dato de posibles sobreestimaciones de los modelos. Respecto a las zonas donde la probabilidad estimada de ocurrencia de incendios es mayor, éstas se corresponden en Zamora con áreas forestales ocupadas por bosques de frondosas, seguidas de mosaicos de vegetación natural arbolada y matorral con herbáceas y de los pastos. En Madrid, se ubican sobre los pastos y los bosques de frondosas.

La validación independiente con los datos de 2011 mostró que la mayoría de los incendios (>80\%) se originaron en zonas de probabilidad media en el modelo de interfaces generales. En el modelo de interfaces específicas, el $41 \%$ de los incendios del año 2011 de Zamora y Madrid se iniciaron en zonas de probabilidad baja. Estos resultados pueden deberse a que los datos de un solo año pueden no ser significativos, por lo que hacerlo con una serie temporal más larga podría mejorar la validación.

A pesar de las bondades del método, MaxEnt presenta una limitación: el resultado del análisis puede estar influido por algunas zonas del área de estudio que tienen mayor abundancia de incendios (Zadrozny 2004, Phillips et al. 2009, Vilar et al. 2016). Por su parte, Parisien y Moritz (2009) demostraron que los modelos como MaxEnt, basados en datos de presencia (y no ausencia) de la variable dependiente, presentan mejores resultados cuando una amplia porción del territorio de estudio no se quema pero tiene una alta probabilidad de sufrir un incendio, como podría ser el caso de Madrid, territorio en el que no se producen numerosos incendios pero que tiene un riesgo elevado de sufrirlos debido a la presión humana que se da en el mismo. Los modelos no paramétricos como MaxEnt funcionan mejor que un GLM (Vilar et al. 2016) en el caso de variables que no tienen una distribución estadística conocida a priori, como puede ser el caso de las variables espaciales de interfaz. La distribución de las probabilidades, sin embargo, depende más de las variables (interfaces) que de la relación idoneidad (verdaderos positivos)-error de omisión (falsos negativos) (Vilar et al. 2016).

La incorporación de otras variables de tipo socio-económico (carreteras, ferrocarriles, población, etc.) podría contribuir a mejorar los ajustes, completando el conjunto de factores explicativos de la ocurrencia vinculada a factores socio-económicos. Sin embargo, algunas de esas variables son difíciles de obtener a la escala o el nivel de actualización necesarios, por lo que es relevante conocer el grado de ajuste que se puede obtener con variables relacionadas, exclusivamente, con el uso y cobertura del suelo. En futuros estudios se planteará la incorporación de variables biofísicas de tipo climático (temperatura, precipitación), topográfico (pendiente, orientación) o relacionadas con el tipo y estado del combustible para completar el conjunto de variables explicativas y mejorar los ajustes globales del modelo.

\section{Conclusiones}

La modelización de la ocurrencia de incendio forestal vinculada a las actividades humanas es de gran interés debido a que, en nuestro entorno mediterráneo, más del $80 \%$ de los incendios se deben a causa humana. Representar este factor humano reviste complejidad dada la gran cantidad de actividades e interacciones que se producen en un territorio. Los resultados de 


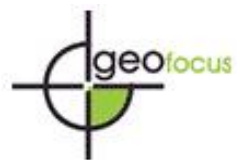

Garrido, J., Vilar, L., Echavarría, P., Martínez-Vega, J, Martín M. P. (2018): “¿Pueden las interfaces de usos del suelo explicar la ocurrencia de incendios forestales a escala provincial? Los casos de Zamora y Madrid", GeoFocus (Artículos), no 22, p. 71-95. ISSN: 1578-5157 http://dx.doi.org/10.21138/GF.611

este trabajo demuestran que es posible obtener modelos predictivos de ocurrencia de incendios a escala provincial a partir de variables de interfaz que representan actividades humanas que se desarrollan en estas zonas y que son susceptibles de dar lugar a un incendio forestal. En este trabajo se han generado modelos mediante el método MaxEnt (algoritmo genético) en dos provincias españolas (Zamora y Madrid) con distintas características territoriales socioeconómicas y ambientales. Se han generado modelos con interfaces generales (agrícola-forestal, urbano-forestal y pasto-forestal) y se han comparado con otros obtenidos a partir de interfaces específicas, desglosando las categorías generales (pasto, forestal y agrícola) en otras más detalladas según la clasificación disponible (caducifolias, frondosas, matorral, coberturas mixtas, etc.). El empleo de interfaces específicas ha producido un incremento en el ajuste general de los modelos y ha proporcionado una mejora en la sensibilidad y disminución de errores en la provincia de Zamora. Además, los modelos específicos han aportado valores más altos de probabilidad de ocurrencia lo que puede ser relevante de cara a las labores de prevención y gestión contra incendio. Las variables explicativas de los distintos modelos (interfaces) han sido obtenidas a partir de una fuente de datos global, homogénea y actualizada (mapa CCI-LC), lo que permitiría la aplicación de los modelos propuestos a otras zonas de estudio y momentos temporales.

\section{Agradecimientos}

El presente estudio se ha desarrollado en el marco del proyecto LUC4FIRE de la convocatoria de Jóvenes Investigadores (JIN) del MINECO (CSO2015-73407-JIN) y gracias al programa de Garantía Juvenil de la Comunidad de Madrid (CAMPD_MAD_CCHS_001). Agradecemos a la Dirección General del Medio Natural de la Consejería de Fomento y Medio Ambiente de la Junta de Castilla y León y al Cuerpo de Bomberos de la Comunidad de Madrid (Dirección General de Protección Ciudadana. Consejería de Presidencia, Justicia y Portavocía del Gobierno. Comunidad de Madrid) el acceso a los datos de ocurrencia de incendios de Zamora y Madrid.

\section{Bibliografía}

Agencia Estatal de Meteorología, A.E.M.E.T. (2011): Atlas Climático Ibérico. Elaborado por el Departamento de Producción de AEMET y por el Departamento de Meteorología y Clima del Instituto de Meteorología de Portugal. Ministerio de Agricultura, Alimentación y Medio Ambiente. pp. 1-80.

Baldwin, R.B. (2009): Use of Maximum Entropy Modelling in Wildlife Research. Entropy, 11, pp. 854-866.

Bar Massada, A., Radeloff, V.C., Stewart, S.I.,Hawbaker, T.J. (2009): Wildfire risk in the wildland-urban interface: A simulation study in northwestern Wisconsin. Forest Ecology and Management, 258, pp. 1990-1999.

Bar Massada, A., Syphard, A.D., Stewart, S.I.,Radeloff, V.C. (2012): Wildfire ignitiondistribution modelling: a comparative study in the Huron-Manistee National Forest, Michigan, USA. International Journal of Wildland Fire, 22, pp. 174-183.

Bond, W.J.,van Wilgen, B.W. (1996): Fire and Plants. Population and community biology series, 14, pp. 1-227. 
Garrido, J., Vilar, L., Echavarría, P., Martínez-Vega, J, Martín M. P. (2018): “¿Pueden las interfaces de usos del suelo explicar la ocurrencia de incendios forestales a escala provincial? Los casos de Zamora y Madrid", GeoFocus (Artículos), no 22, p. 71-95. ISSN: 1578-5157 http://dx.doi.org/10.21138/GF.611

Bowman, D.M., Balch, J., Artaxo, P., Bond, W.J., Cochrane, M.A., D'Antonio, C.M., Defries, R., Johnston, F.H., Keeley, J.E., Krawchuk, M.A., Kull, C.A., Mack, M., Moritz, M.A., Pyne, S., Roos, C.I., Scott, A.C., Sodhi, N.S., Swetnam, T.W.,Whittaker, R. (2011): The human dimension of fire regimes on Earth. Journal of Biogeography, 38(12), pp. 2223-2236.

Camarero, L. (1993): Del éxodo rural y del éxodo urbano. Ocaso y Renacimiento de los asentamientos rurales en España. Madrid: Ministerio de Agricultura, Pesca y Alimentación, pp. $1-524$.

Cohen, J.D. (2000): Preventing disaster: home ignitability in the wildland-urban interface. Journal of Forestry, 98, pp. 15-21.

Comunidad de Madrid (2007): Atlas. El Medio Ambiente de la Comunidad de Madrid. http://www.madrid.org/bvirtual/BVCM003345.pdf (consultado 25-04-2018).

Castilla y León (2018). Consejería de Fomento y Medio Ambiente de Castilla y León. http://www.medioambiente.jcyl.es/ (consultado 25-04-2018).

Couturier, T., Besnard, A., Bertolero, A., Bosc, V., Astruc, G.,Cheylan, M. (2014): Factors determining the abundance and occurrence of Hermann's tortoise Testudo hermanni in France and Spain: fire regime and landscape changes as the main drivers. Biological Conservation, 170, pp. 177-187.

Cubo, J.E., Enríquez, E., Gallar, J.J., López. M., Mateo, M.L., Muñoz, A.,Parra, P.J. (2012): Los Incendios Forestales en España. Decenio 2001-2010. Ministerio de Agricultura, Alimentación y Medio Ambiente, M.A.P.A.M.A., pp. 1-138.

Chou, Y.H., Minnich, R.A., Chase, R.A. (1993): Mapping probability of fire occurrence in San Jacinto Mountains, California, USA. Environmental Management, 17, pp. 129-140.

Chuvieco, E., Aguado, I., Yebra, M., Nieto, H., Salas, J., Martín, M.P., Vilar, L., Martínez, J., Martín, S., Ibarra, I., De La Riva, J., Baeza, J., Rodríguez, F., Molina, J.R., Herrera, M.A.,Zamora, R. (2010): Development of a framework for fire risk assessment using remote sensing and geographic information system technologies. Ecological Modelling, 221, pp. 46-58.

Deblauwe, V., Barbier, N., Couteron, P., Lejeune, O.,Bogaert, J. (2008): The global biogeography of semi-arid periodic vegetation patterns. Global Ecology and Biogeography, 17(6), pp. 715-723.

Defourny, P., Santoro, M., Kirches, G., Wevers, J., Boettcher, M., Brockmann, C., Lamarche, C.,Bontemps, S. (2017): Land Cover CCI Product User Guide Version 2.0. UCL-Geomatics, Louvain-la-Neuve, Belgium, pp. 1-105.

Diputación de Zamora (2018). http://www.diputaciondezamora.es (consultado 25-04-2018).

Egan, J.P. (1975): Signal detection theory and ROC analysis. Series in Cognition and Perception. Academic Press, New York., pp. 1-277.

Elith, J., Graham, C.H., Anderson, R.P., Dudik, M., Ferrier, S., Guisan, A., Hijmans, R.J., Huettmann, F., Leathwick, J.R., Lehmann, A., Li, J., Lohmann, L.G., Loiselle, B.A., Manion, G., Moritz, C., Nakamura, M., Nakazawa, Y., Overton, J.M., Peterson, A.T., Phillips, S.J., Richardson, K.S., Scachetti-Pereira, R., Schapire, R.E., Soberón, J., Williams, S., Wisz, M.S.,Zimmermann, N.E. (2006): Novel methods improve prediction of species' distributions from occurrence data. Ecography, 29(2), pp. 129-151.

Elith, J., Kearney, M.,Phillips, S. (2010): The art of modelling range-shifting species. Methods in Ecology and Evolution, 1, pp. 330-342. 
Garrido, J., Vilar, L., Echavarría, P., Martínez-Vega, J, Martín M. P. (2018): “¿Pueden las interfaces de usos del suelo explicar la ocurrencia de incendios forestales a escala provincial? Los casos de Zamora y Madrid", GeoFocus (Artículos), no 22, p. 71-95. ISSN: 1578-5157 http://dx.doi.org/10.21138/GF.611

ESRI. (2014): Environmental System Research Institute (ESRI). ArcGIS Desktop Help 10.2. Geostatistical Analyst, Standfor, CA, USA, pp.

Feng, C., Yongsheng, D., Shukui, N.Jinlong, Z. (2015): Modeling Forest Lightning Fire Occurrence in the Daxinganling Mountains of Northeastern China with MAXENT. Forests, 6 , pp. 1422-1438.

Gallardo, M., Gómez, I., Vilar, L., Martínez-Vega, J.,Martín, M.P. (2015): Impacts of future land use/land cover on wildfire occurrence in the Madrid region (Spain). Regional Environmental Change, 16(4), pp. 1047-1061.

Ganteaume, A., Camia, A., Jappiot, M., San-Miguel-Ayanz, J., Long-Fournel, M.,Lampin, C. (2013): A Review of the Main Driving Factors of Forest Fire Ignition Over Europe. Environmental Management, 51, pp. 651-662.

Giovannini, A., Seglie, D.,Giacoma, C. (2014): Identifying priority areas for conservation of spadefoot toad, Pelobates fuscus insubricus using a maximum entropy approach. Biodiversity and Conservation, 23, pp. 1427-1439.

Guisan, A., Graham, C.H., Elith, J.,Huettmann, F. (2007): Sensitivity of predictive species distribution models to change in grain size. Diversity and Distributions, 13(3), pp. 332-340.

Gutiérrez, J.M., Bedia, J., Cofiño, A.S., Fernández, J.,San-Martín, D. (2013): Forest fires under climate, social and economic changes in Europe, the Mediterranean and other fire-affected areas of the world. European Project http://fumeproject.uclm.es/, pp. 1-32.

Hanley, J.,McNeil, B. (1982): The meaning and use of the area under a receiver operating characteristic (ROC) curve. Radiology, 143, pp. 29-36.

Hastie, T.J., Tibshirani, R.J.,Friedman, J. (2009): The elements of statistical learning. Dordrecht, editor: Springer, pp. 1-763.

INE (2018): INEbase. Estadísticas territoriales. http://www.ine.es/FichasWeb/RegProvincias.do (consultado 25-04-2018).

Lampin-Maillet, C., Long-Fournel, M., Ganteaume, A., Jappiot, M.,Ferrier, J.P. (2011): Land cover analysis in wildland-urban interfaces according to wildfire risk: A case study in the South of France. Forest Ecology and Management, 261(12), pp. 2200-2213.

Leone, V., Koutsias, N., Martínez, J., Vega-García, C., Allgöwer, B.,Lovreglio, R. (2003): The Human Factor in Fire Danger Assessment. In: Chuvieco, E., editor. Wildland Fire Danger Estimation and Mapping The Role of Remote Sensing Data. Singapore: World Scientific Publishing., 4, pp. 143-194.

Martínez, J., Vega-García, C.,Chuvieco, E. (2009): Human-caused wildfire risk rating for prevention planning in Spain. Journal of Environmental Management, 90(2), pp. 1241-1252.

MaxEnt. Biodiversity Informatics. American Museum of Natural History. https://biodiversityinformatics.amnh.org/open_source/MaxEnt/ (consultado 25-04-2018)

Millington, J.D.A., Wainwright, J., Perry, G.L.W., Romero-Calcerrada, R.,Malamud, B.D. (2009): Modelling Mediterranean landscape succession-disturbance dynamics: A landscape firesuccession model. Environmental Modelling \& Software, 24(10), pp. 1196-1208.

Nafría, D.A., Garrido, N., Álvarez, M.V., Cubero, D., Fernández, M., Villarino, I., Gutiérrez, A.,Abia, I. (2013): Atlas Agroclimático de Castilla y León. Junta de Castilla y León. Instituto 
Garrido, J., Vilar, L., Echavarría, P., Martínez-Vega, J, Martín M. P. (2018): “¿Pueden las interfaces de usos del suelo explicar la ocurrencia de incendios forestales a escala provincial? Los casos de Zamora y Madrid", GeoFocus (Artículos), no 22, p. 71-95. ISSN: 1578-5157 http://dx.doi.org/10.21138/GF.611

Tecnológico Agrario de Castilla y León. Ministerio de Agricultura, Alimentación y Medio Ambiente., pp. 1-137.

National Interagency Fire Center, N.I.F.C. (2004): Wildland fire statistics. pp.

Nelder, J.A.,Wedderburn, R.W.M. (1972): Generalized Linear Models. Journal of the Royal Statistical Society. Series A (General), 135(3), pp. 370-384.

Nicolás, J.M.,Caballero, D. (2001): Demanda territorial de defensa contra incendios forestales. Un caso de estudio: Comunidad de Madrid. Proceedings of Spanish National Forest Congress. Granada 2001, pp. 1-16.

Oliveira, S., Oehler, F., San-Miguel-Ayanz, J., Camia, A.,Pereira, J.M.C. (2012): Modeling spatial patterns of fire occurrence in Mediterranean Europe using Multiple Regression and Random Forest. Forest Ecology and Management, 275(0), pp. 117-129.

Padilla, M.,Vega-García, C. (2011): On the comparative importance of fire danger rating indices and their integration with spatial and temporal variables for predicting daily human-caused fire occurrences in Spain. International Journal of Wildland Fire, 20, pp. 46-58.

Parisien, M.A.,Moritz, M.A. (2009): Environmental controls on the distribution of wildfire at multiple spatial scales. Ecological Monographs, 79, pp. 127-154.

Parisien, M.A., Snetsinger, S., Greenberg, J.A., Nelson, C.R., Schoennagel, T., Dobrowski, S.Z.,Moritz, M.A. (2012): Spatial variability in wildfire probability across the western United States. International Journal of Wildland Fire, 21, pp. 313-327.

Pearson, R.G., Raxworthy, C.J., Nakamura, M.,Peterson, A.T. (2007): Predicting species distributions from small numbers of occurrence records: A test case using cryptic geckos in Madagascar. Journal of Biogeography, 34(1), pp. 102-117.

Pena, J.C.C., Kamino, L.H.Y., Rodrigues, M., Mariano-Neto, E.,Siqueira, M.F. (2014): Assessing the conservation status of species with limited available data and disjunct distribution. Biological Conservation, 170, pp. 130-136.

Pérez-Cabello, F., Echevarría, M.T., Ibarra, P.,de la Riva, J. (2009): Effects of Fire on Vegetation, Soil and Hydrogeomorphological Behaviour in Mediterranean Ecosystems. In: Chuvieco E, editor. Earth Observation of Wildland Fires in Mediterranean Ecosystems., pp. 111128.

Phillips, S.J., Anderson, R.P.,Schapire, R.E. (2006): Maximum entropy modeling of species geographic distributions. Ecological Modelling, 190, pp. 231-259.

Phillips, S.J., Dudik, M., Elith, J., Graham, C.H., Lehmann, A., Leathwick, J.,Ferrier, S. (2009): Sample selection bias and presence-only distribution models: implications for background and pseudo-absence data. Ecological Applications, 19(1), pp. 187-197.

Phillips, S.J., Dudik, M.,Schapire, R.E. (2004): A Maximum Entropy Approach to Species Distribution Modeling. Proceedings of the Twenty-First International Conference on Machine Learning, pp. 655-662.

Radeloff, V.C., Hammer, R.B., Stewart, S.I., Fried, J.S., Holcomb, S.S.,McKeefry, J.F. (2005): The Wildland-Urban Interface in the United States. Ecological Applications, 15(3), pp. 799-805.

Renard, Q., Pélissier, R., Ramesh, B.R.,Kodandapani, N. (2012): Environmental susceptibility model for predicting forest fire occurrence in the Western Ghats of India. International Journal of Wildland Fire, 21, pp. 368-379. 
Garrido, J., Vilar, L., Echavarría, P., Martínez-Vega, J, Martín M. P. (2018): “¿Pueden las interfaces de usos del suelo explicar la ocurrencia de incendios forestales a escala provincial? Los casos de Zamora y Madrid", GeoFocus (Artículos), no 22, p. 71-95. ISSN: 1578-5157 http://dx.doi.org/10.21138/GF.611

Rodrigues, M., de la Riva, J.,Fotheringham, S. (2014): Modeling the spatial variation of the explanatory factors of human-caused wildfires in Spain using geographically weighted logistic regression. Applied Geography, 48, pp. 52-63.

Rodríguez-Rodríguez, D., Martínez-Vega, J. (2017). Assessing recent environmental sustainability in the Spanish network of National Parks and their statutory peripheral areas. Applied Geography, 89, pp. 22-31.

Romero-Calcerrada, R., Novillo, C.J., Millington, J.D.A.,Gómez-Jiménez, I. (2008): GIS analysis of spatial patterns of human-caused wildfire ignition risk in the SW of Madrid (Central Spain). Landscape Ecology, 23, pp. 341-354.

Romero-Calcerrada, R.,Perry, G.L.W. (2004): The role of land abandonment in landscape dynamics in the SPA 'Encinares del río Alberche y Cofio, Central Spain, 1984-1999. Landscape and Urban Planning, 66, pp. 217-232.

Spessa, A., McBeth, B.,Prentice, C. (2005): Relationships among fire frequency, rainfall and vegetation patterns in the wet-dry tropics of northern Australia: an analysis based on NOAAAVHRR data. Global Ecology and Biogeography, 14, pp. 439-454.

Sturtevant, B.R.,Cleland, D.T. (2007): Human and biophysical factors influencing modern fire disturbance in northern Wisconsin. International Journal of Wildland Fire, 16, pp. 398-413.

Syphard, A.D., Radeloff, V.C., Keeley, J.E., Hawbaker, T.J., Clayton, M.K., Stewart, S.I.,Hammer, R.B. (2007): Human influence in California Fire regimes. Ecological Applications, 17(5), pp. 1388-1402.

Syphard, A.D., Radeloff, V.C., Keuler, N.S., Taylor, R.S., Hawbaker, T.J., Stewart, S.I.,Clayton, M.K. (2008): Predicting spatial patterns of fire on a southern California landscape. International Journal of Wildland Fire, 17, pp. 602-613.

van der Werf, G.R., Randerson, J.T., Collatz, G.J.,Giglio, L. (2003): Carbon emissions from fires in tropical and subtropical ecosystems. Global Change Biology, 9, pp. 547-562.

Vilar, L., Gómez, I., Martínez-Vega, J., Echavarría, P., Riaño, D.,Martín, M.P. (2016): Multitemporal Modelling of Socio-Economic Wildfire Drivers in Central Spain between the 1980s and the 2000s: Comparing Generalized Linear Models to Machine Learning Algorithms. PLOS ONE, 11(8), pp. 1-17.

Vilar, L., Martín, M.P.,Martínez, F.J. (2008): Empleo de técnicas de regresión logística para la obtención de modelos de riesgo humano de incendio forestal a escala regional. Boletín de la $A G E, 47$, pp. 5-29.

Vilar, L., Martín, M.P.,Martínez, F.J. (2011): Logistic regression models for human-caused wildfire risk estimation: analysing the effect of the spatial accuracy in fire occurrence data. European Journal of Forest Research, 130, pp. 983-996.

Vilar, L., Woolford, D.G., Martell, D.L.,Martín, M.P. (2010): A model for predicting humancaused wildfire occurrence in the region of Madrid, Spain. International Journal of Wildland Fire, 19, pp. 325-337.

Winter, G.J.,Fried, S.J. (2001): Estimating contingent values for protection from wildland fire using a two-stage decision framework. Forest Science, 47(3), pp. 349-360.

Zadrozny, B. (2004): Learning and evaluating classifiers under sample selection bias. Proceedings of the twenty-first international conference on Machine learning. Banff, Alberta, Canada. 1015425: ACM., pp. 114. 
